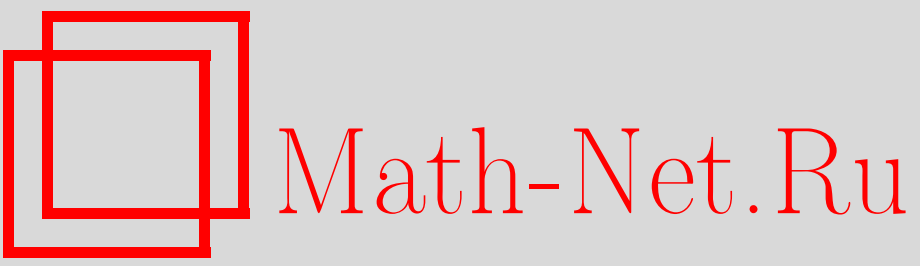

О. И. Богоявленский, Точные глобальные равновесия плазмы, УМН, 2000, том 55, выпуск 3, 63-102

DOI: https://doi.org/10.4213/rm291

Использование Общероссийского математического портала Math-Net.Ru подразумевает, что вы прочитали и согласны с пользовательским соглашением

http://www.mathnet.ru/rus/agreement

Параметры загрузки:

IP : 52.90 .164 .192

26 апреля 2023 г., 16:40:30 


\title{
ТОЧНЫЕ ГЛОБАЛЬНЫЕ РАВНОВЕСИЯ ПЛАЗМЫ
}

\author{
О. И. БОГОявЛЕНСКИЙ
}

\begin{abstract}
Выведены точные глобальные равновесия плазмы, обладающие осевой и винтовой симметриями. Эти два семейства точных решений уравнений равновесия плазмы не являются трансляционно-инвариантными, зависят от произвольно болшого числа параметров и содержат специальные $z$-инвариантные равновесия. Все построенные равновесия плазмы являются гладкими и локализованными в том смысле, что они имеют конечную магнитную энергию в каждом слое $c_{1}<z<c_{2}$. Кроме того, эти точные решения доставляют контрпримеры к известной теореме Паркера.

Библиография: 60 названий.
\end{abstract}

\section{СОДЕРЖАНИЕ}

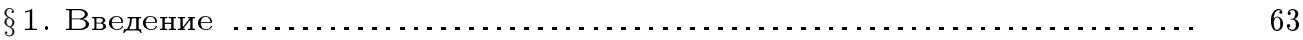

Часть І. Глобальные осесимметричные равновесия плазмы ............ 69

$\S 2$. Нетрансляционно-инвариантные глобальные равновесия плазмы ... 69

§3. Примеры квазипериодических и периодических равновесий плазмы . 72

$\S 4$. Квазипериодичность глобальных равновесий плазмы ............. 76

$\S 5$. Глобальные равновесия плазмы в полупространстве .............. 78

$\S 6$. Контрпримеры к теореме Паркера ............................ 79

Часть II. Равновесия плазмы с винтовой симметрией .................. 83

$\S 7$. Точные глобальные равновесия плазмы с винтовой симметрией $\ldots . . .83$

$\S 8$. Модель астрофизических струй ................................ 89

$\S 9$. Квазипериодичность магнитных силовых линий $\ldots \ldots \ldots \ldots \ldots \ldots \ldots \ldots . . . \ldots 2$

§10. Винтовые равновесия плазмы, зависящие от $2 K+1$ параметров .... 95

$\S 11$. Контрпримеры к теореме Паркера, обладающие винтовой симмет-

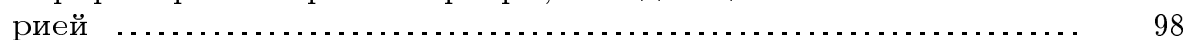

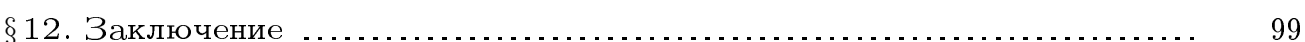

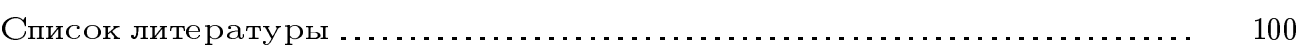

\section{$\S 1$. Введение}

I. В данном обзоре представлены математические результаты автора по глобальным равновесиям плазмы и контрпримеры к известной теореме Паркера, опубликованные недавно в кратком виде в физических журналах [1]-[3]. Поиск глобальных 
равновесий плазмы, удовлетворяюших уравнениям

$$
\mathbf{J} \times \mathbf{B}=\operatorname{grad} p, \quad \operatorname{div} \mathbf{B}=0, \quad \mathbf{J}=\frac{1}{\mu} \operatorname{curl} \mathbf{B},
$$

продолжался в течение последних сорока лет, с тех пор как уравнения (1.1) были впервые применены к управляемому термоядерному синтезу [4]-[7] и к астрофизическим проблемам [8], [9]. Однако все найденные до сих пор точные решения уравнений (1.1), не являюшиеся трансляционно-инвариантными, или имеют сингулярности, или неограниченно растут на бесконечности [10]-[14], или не являются локализованными [15]-[17]. Такие решения имеют весьма ограниченную применимость в астрофизике. Здесь $\mathbf{B}$ - магнитное поле, $\mathbf{J}$ - плотность электрического тока, $p$ - давление плазмы и $\mu$ - магнитная проницаемость среды.

Как было отмечено впервые Ландквистом [18], уравнения (1.1) эквивалентны уравнениям равновесия идеальной несжимаемой жидкости

$$
\mathbf{V} \times \operatorname{curl} \mathbf{V}=\operatorname{grad}\left(\frac{p}{\rho_{0}}+\frac{V^{2}}{2}\right), \quad \operatorname{div} \mathbf{V}=0 .
$$

Поэтому поиск глобальных решений уравнений (1.1) важен также для гидродинамики.

Классическая теорема Праудмана утверждает, что любое малое возмушение $z$-инвариантного однородного врашения идеальной несжимаемой жидкости также является $z$-инвариантным. Эта теорема была доказана Праудманом [19] и Тейлором [20] и позже подтверждена Тейлором экспериментально [21]. Теорема Праудмана была обобщена Паркером [22], [23] для уравнений равновесия плазмы (1.1).

II. Первоочередной целью данного обзора является представление новых точных решений уравнений равновесия плазмы, которые моделируют астрофизические струи в сопутствуюшей системе отсчета и солнечные протуберанщы [25]. Такие равновесия должны быть глобальными, что означает, что они должны удовлетворять следующим необходимым физическим условиям:

a) магнитное поле $\mathbf{B}$, ток $\mathbf{J}$ и давление плазмы $p$ являются гладкими и ограниченными функциями всюду в евклидовом пространстве $\mathbb{R}^{3}$;

b) при $r \rightarrow \infty$ магнитное поле $\mathbf{B} \rightarrow 0$, плотность тока $\mathbf{J} \rightarrow 0$, давление $p \rightarrow p_{1}$;

c) все магнитные силовые линии и линии тока являются ограниченными по радиальной переменной $r$ в цилиндрических координатах $r, z, \phi$.

В ч. I мы выводим два семейства точных глобальных осесимметричных равновесий плазмы, которые зависят от произвольного числа свободных параметров. Первое семейство определено во всем евклидовом пространстве $\mathbb{R}^{3}$, второе определено в полупространстве $z \geqslant 0$. Эти точные равновесия плазмы моделируют многообразие магнитных полей, наблюдаемых в астрофизических струях и в солнечной короне [25]. Асимптотическое значение давления $p_{1}$ в условии b) является средним давлением в астрофизическом истекающем потоке и в солнечной корональной плазме. Как обычно, гравитационная сила $-\rho \operatorname{grad} \Psi$ включается в градиент давления в $(1.1)$, в приближении постоянной плотности $\rho$. Равновесия плазмы являются локализованными в том смысле, что полная магнитная энергия в любом слое $c_{1}<z<c_{2}$ является конечной. 
Для решений второго семейства полная магнитная энергия в полупространстве $z \geqslant 0$ является конечной.

Общие введенные равновесные решения в $\mathbb{R}^{3}$ являются квази-периодическими по переменной $z$ с $N-1$ частотами, пропорциональными числам

$$
1, \sqrt{2}, \sqrt{3}, \ldots, \sqrt{N-1}
$$

с общим множителем $\sqrt{8 \beta}$. Доказано, что в последовательности (1.3) имеется приближенно $6 \mathrm{~N} / \pi^{2}$ рационально независимых квадратных корней. Поэтому магнитное поле $\mathbf{B}$ и ток $\mathbf{J}$ действительно являются квази-периодическими по переменной $z$.

Замечательно, что классическая теоретико-числовая проблема о ранге последовательности (1.3) над рациональными числами возникла из изучения глобальных равновесий плазмы. Эта проблема является естественным расширением теоремы Пифагора об иррациональности $\sqrt{2}[26]$.

Другим важным свойством полученных точных решений является то, что они образуют $(2 N-1)$-мерные линейные пространства. Любая линейная комбинация соответствуюших магнитных полей В снова является точным решением. Однако закон сложения давления плазмы $p$ имеет более сложный квадратичный вид. Эти свойства напоминают свойства $N$-солитонных решений интегрируемых уравнений физики плазмы: уравнений КдФ, КП и НШ [27], [28].

Первым из построенных точных решений является гауссово распределение $\mathbf{B}=$ $\exp \left(-\beta r^{2}\right) \widehat{\mathbf{e}}_{z}$. Гауссово распределение играет решаюшую роль в выведенных глобальных равновесиях плазмы (1.1). Решения убывают при $r \rightarrow \infty$ так же быстро, как $c_{N} \exp \left(-\beta r^{2}\right) r^{2 N}$, из чего следует сильная фокусировка астрофизической струи.

Точное решение $\mathbf{B}=\exp \left(-\beta r^{2}\right) \widehat{\mathbf{e}}_{z}, \mathbf{J}=4 \beta^{2} r \exp \left(-\beta r^{2}\right) \widehat{\mathbf{e}}_{\phi} / \mu$ имеет геометрию $\theta$-пинча. Другие точные решения с цилиндрической симметрией имеют геометрию винтового пинча; их магнитные поверхности являются цилиндрами $r=$ const. Для точных транслящионно-неинвариантных равновесий плазмы магнитные поверхности являются либо цилиндрами, либо вложенными торами с круговыми магнитньми осями. Распределение этих тороидальных магнитных поверхностей является квазипериодическим по переменной $z$. Магнитные оси зацеплены с замкнутыми магнитными силовыми линиями в соответствуюших тороидальных областях.

Выведенные точные равновесия плазмы доставляют семейство динамических систем в $\mathbb{R}^{3}$, определяюших динамику магнитных силовых линий. Эти динамические системы являются интегрируемьми в широком смысле работы [29]. Общие магнитные силовые линии или являются квазипериодическими обмотками торов $\mathbb{T}^{2}$, или движутся на цилиндрах $\mathbb{R}^{1} \times S^{1}$.

III. В ч. II данного обзора мы изучаем равновесия плазмы с винтовой симметрией. Существуют по крайней мере три основных физических явления, связанных с винтовыми конфигурациями плазмы: 1) внешние и внутренние кинк-моды в токамаках, 2) астрофизические струи, 3) взрывные солнечные протуберанцы. Внешние кинк-моды известны как наиболее опасные магнитогидродинамические неустойчивости, возникающие в плазменном пинче, несушем продольньй ток. Внутренняя $(m=1)$ кинковая неустойчивость ассоциируется с винтовым возмушением плазмы, которое предшествует пилообразным колебаниям [30], [31] около магнитной оси токамака. Наилучшее 
аналитическое описание внешних и внутренних кинк-мод для "прямого токамака" доставляется равновесиями плазмы с винтовой симметрией. Эти решения были использованы в численном исследовании пинчей “обратного поля" [32].

Как известно, оптические и радиоастрономические наблюдения открыли многообразие струй в активных ядрах галактик, см. обзор [33]. Двусторонние струи моделировались автомодельными осесимметричньми равновесиями плазмы [34]. Имеется также статистически значимое количество односторонних струй, характерным примером которых является струя в эллиптической галактике М87 в скоплении Вирго. Оптическое излучение этой струи является синхротронным [35]; детектируемьй образ ее интенсивности не имеет осевой симметрии [36]. Мы моделируем эти сильно сконцентрированные струи равновесиями плазмы с винтовой симметрией.

Наблюдения взрьвных солнечных протуберанцев обнаружили трубки плазмы, обладаюшие повьшенной плотностью и винтовой структурой, которые предположительно совпадают со жгутами магнитного потока [37]. Известно, что солнечные протуберанщы могут оставаться в устойчивом равновесии в течение нескольких месяцев.

Все эти конфигурации плазмы с винтовой симметрией описываются уравнением Дж $Ф К О$, выведенным Джонсоном, Фриманом, Калсрудом и Оберманом в работе [38]. Это уравнение является редукцией уравнений равновесия плазмы (1.1) для решений с винтовой симметрией. Уравнение ДжфКО сводится к уравнению Грэда-Шафранова [4], [5], когда винтовая симметрия преврашается в осевую. Оба уравнения широко использовались для моделирования лабораторной плазмы в токамаках, стеллараторах, гелиотронах, торзатронах и астрофизической плазмы.

Мы изучаем решения уравнения ДжфКО [38], которые определены во всем евклидовом пространстве $\mathbb{R}^{3}$ и не имеют сингулярностей. Эти точные равновесия плазмы моделируют многообразие магнитных полей в кинк-модах в токамаках, в астрофизических струях и в солнечной короне. Асимптотическое значение давления $p_{1}$ в условии b) является средним давлением во внешней среде (астрофизический истекающий поток или плазма солнечной короны). Магнитное поле убывает при $r \rightarrow \infty$ так же быстро, как $c_{N} \exp \left(-\beta r^{2}\right) r^{2 N}$. Отсюда следует сильная концентрация астрофизической струи и конечность магнитной энергии в любом слое $c_{1}<z<c_{2}$. Интенсивность синхротронного излучения астрофизической струи пропорциональна $B^{2}[35]$. Поэтому детектируемый образ интенсивности струи определяется формой и распределением поверхностей $B^{2}=$ const.

Для решений с винтовой симметрией магнитные поверхности являются вложенными цилиндрами, которые врашаются как спирали вокруг оси $z$. Наиболее внутренние цилиндры являются их магнитными осями, которые вместе образуют конечную систему спиралей. Простейшее точное решение имеет двойную спираль магнитных осей и моделирует астрофизические струи, плазменную кинк-моду с $m=1$ и закрученные жгуты магнитного потока, наблюдавшиеся в токамаках [10], [12] и в солнечных протуберанщах [37].

Вследствие их винтовой симметрии выведенные точные решения являются периодическими по переменной $z$ с периодом $2 \pi \gamma$. Важной особенностью полученных глобальных равновесий плазмы является то, что их магнитные силовые линии являются в общем случае квазипериодическими в цилиндрических координатах $r, z, \phi$. Это означает, что они никогда не повторяются в $z$-направлении, однако появляются произвольно близко к их начальным данным при изменении переменной $z$. Поэтому об- 
раз врашения магнитных силовых линий вокруг друг друга непрерьвно изменяется в z-направлении для построенных точных глобальных равновесий плазмы.

Мы указываем семейства точных решений с винтовой симметрией, которые образуют размерности $3,5, \ldots$ Любая линейная комбинация соответствующих магнитных полей снова является точным решением. Закон сложения давления плазмы имеет более сложную квадратичную форму. Эти свойства, так же как и в осесимметричном случае, напоминают $N$-солитонные решения интегрируемых уравнений физики плазмы.

Каждое $(2 K+1)$-мерное линейное пространство точных глобальных равновесий плазмы состоит из решений с винтовой симметрией, которые не являются z-инвариантными и являются возмущениями исключительных $z$-инвариантных равновесий. Конструкция этих $(2 K+1)$-мерных пространств точных решений основана на разрешении диофантова уравнения [39]

$$
a x^{2}+2 b x+4 b y=4 b c
$$

по отношению к двум неизвестным целым числам $x$ и $y$, где $a, b, c$ являются произвольными натуральными числами. Число $K+1$ является числом различных пар целых неотрицательных решений $x, y$ диофантова уравнения (1.4).

Поиск глобальных решений уравнений (1.1) важен также для магнитной гидродинамики, поскольку уравнения магнитогидродинамического равновесия

$$
\begin{gathered}
\mathbf{V} \times \operatorname{curl} \mathbf{V}-\mathbf{B} \times \operatorname{curl} \mathbf{B} /(\rho \mu)=\operatorname{grad}\left(w+V^{2} / 2\right), \\
\operatorname{div} \mathbf{V}=0, \quad \operatorname{div} \mathbf{B}=0, \quad[\mathbf{V}, \mathbf{B}]=0
\end{gathered}
$$

эквивалентны уравнениям (1.1) в случае параллельных векторных полей $\mathbf{B}=\lambda \mathbf{V}$ и постоянной плотности $\rho$; энтальпия $w$ удовлетворяет уравнению $d w=d p / \rho$. Поэтому точные решения, представленные в этом обзоре, описывают также глобальные равновесия плазмы с $\mathbf{B}=\lambda \mathbf{V}$, где $\lambda \neq \sqrt{\rho \mu}$.

IV. В качестве вторичной цели я хотел бы отметить, что выведенные точные решения доставляют контрпримеры к широко известной теореме Паркера [22]-[24], которая рассматривает малые возмушения $z$-инвариантных равновесий плазмы и которая обсуждалась в течение более чем 25 лет. В работе [22] Паркер рассматривает специальный случай проблемы: малые возмущения однородного магнитного поля $\mathbf{B}=B_{0} \widehat{\mathbf{e}}_{z}, B_{0}=$ const, $p=$ const. Возмущения общих $z$-инвариантных равновесий изучены в ч. 14 монографии [23]. Паркер пишет (с. 374): "Рассмотрим магнитное поле $B_{i}(x, y)+\varepsilon b_{i}(x, y, z)$ в окрестности общего равновесного поля $B_{i}(x, y)$ ", и после детального исследования приходит к заключению на с. 377 :

“Таким образом, в общем случае мы приходим $к$ заключению, что инвариантность $\partial b_{i} / \partial z=0$ (14.51) является необходимым условием равновесия. Любое поле, в котором характер вращения меняется вдоль поля, так что (14.51) исключается его топологией, не может бить в равновесии". 
Это заключение было названо "теоремой Паркера" в работах [40], [41]. Отсутствие точных глобальных решений уравнений (1.1) сделало невозможной независимую проверку теоремы Паркера. Множество следствий и обобщений были получены в предположении, что теорема верна, см. работы [40]-[48].

Свойства глобальных равновесий плазмы были предметом интересной дискуссии в литературе. В 1985 году Ван Баллегойен представил в работах [49], [50] рассуждение о том, что некоторые нетранслящионно инвариантные возмущения (зависящие от $z$ ) могут быть построены с помощю разложения в степенные ряды и последовательного разрешения некоторой бесконечной системы уравнений в частных производных. Однако это рассуждение оказалось неубедительным, поскольку ни отсутствие сингулярностей в решениях бесконечной системы уравнений в частных производных не было доказано (в принципе, могут появиться каустики), ни радиус сходимости степенных рядов не был оценен (он может быть нулем или конечным числом). В дополнение к этому никаких точных решений не было найдено. Поэтому Виллата и Цинганос написали в 1993 году в работе [47; с. 2158], в полном противоречии с результатами Ван Баллегойена и в полном согласии с теоремой Паркера:

"Широко известно, что все хорошо ведущие себя МГД-равновесия, продолжсающиеся во все пространство, обязаны быть транслячионно инвариантными".

Результаты данного обзора проливают некоторый свет на эту дискуссию. Действительно, мы получили точные равновесия плазмы, которые являются гладкими и ограниченными во всем евклидовом пространстве. Эти равновесия не имеют поверхностных токов и не имеют разрывов и удовлетворяют вышеуказанньм физическим условиям a), b), c). Эти точные решения также удовлетворяют всем условиям Паркера [23; с. 359-391]. В $\S 6$ мы строим семейство глобальных $z$-инвариантных равновесий плазмы, причем каждое $N$-е равновесие обладает $(2 N-1)$-мерным линейным пространством глобальных возмушений. Наиболее важньм свойством этих точных решений является то, что они зависят от переменной $z$ и поэтому они не являются $z$-инвариантньми. Эти осесимметричные контрпримеры доказывают, что теорема Паркера не верна. В $\S 6$ мы указываем один из источников расхождения с результатами Паркера в ч. 14 монографии [23].

Следствием z-квазипериодичности полученных равновесных решений является то, что они очень далеки не только от $z$-инвариантности, но даже и от $z$-периодичности. Вследствие z-квазипериодичности “характер вращения" магнитных силовых линий непрерьвно меняется вдоль оси $z$ и не повторяется.

В $\S 11$ мы строим семейство глобальных z-инвариантных равновесий плазмы, причем каждое равновесие обладает трехмерным линейным пространством глобальных возмушений с винтовой симметрией. Эти точные решения также зависят от переменной $z$, и поэтому они не являются $z$-инвариантными. Они удовлетворяют всем условиям Паркера [23; с. 359-391] и указанным вьше физическим условиям а), b), с). Поэтому эти глобальные равновесия плазмы с винтовой симметрией образуют второе семейство контрпримеров к теореме Паркера. 
Часть І. Глобальные осесимметричные равновесия плазмы

\section{§ 2. Нетрансляционно-инвариантные глобальные равновесия плазмы}

I. Для получения точных равновесий плазмы мы рассмотрим уравнения (1.1) для осесимметричных магнитных полей В [4]-[6]:

$$
\mathbf{B}=\frac{\psi_{z}}{r} \widehat{\mathbf{e}}_{r}-\frac{\psi_{r}}{r} \widehat{\mathbf{e}}_{z}+\frac{I}{r} \widehat{\mathbf{e}}_{\phi},
$$

где $\psi(r, z)$ есть функция магнитного потока, $\psi_{x}=\partial \psi / \partial x$ и векторы $\widehat{\mathbf{e}}_{r}, \widehat{\mathbf{e}}_{z}, \widehat{\mathbf{e}}_{\phi}$ являются координатными единичными ортами. Плотность потока $\mathbf{J}$ имеет вид

$$
\mathbf{J}=\frac{1}{\mu} \operatorname{curl} \mathbf{B}=-\frac{I_{z}}{\mu r} \widehat{\mathbf{e}}_{r}+\frac{I_{r}}{\mu r} \widehat{\mathbf{e}}_{z}+\frac{\Phi}{\mu r} \widehat{\mathbf{e}}_{\phi},
$$

где $\Phi=\psi_{r r}-r^{-1} \psi_{r}+\psi_{z z}$. Уравнения равновесия плазмы (1.1) для осесимметричных решений эквивалентны равенствам $I=I(\psi), p=p(\psi)$ и уравнению Грэда-Шафранова [4], [5], выведенному в 1958 году:

$$
\psi_{r r}-\frac{1}{r} \psi_{r}+\psi_{z z}+I(\psi) I^{\prime}(\psi)+\mu r^{2} p^{\prime}(\psi)=0,
$$

где $I(\psi)$ и $p(\psi)$ являются произвольньми гладкими функциями. Из уравнения $(2.3)$ следует $\Phi=-I(\psi) I^{\prime}(\psi)-\mu r^{2} p^{\prime}(\psi)$. Для уравнений равновесия жидкости (1.2) peдукция к уравнению (2.3) была получена Брэггом и Хауфорном в 1950 году [51].

Tеорема 1. Существует бесконечное семейство z-инвариантных глобальных равновесий плазмы, зависящих от челого числа $N \geqslant 0$ и вещественного числа $\beta>0$. Для $N \geqslant 2$ каждое из этих равновесий содержится в $(2 N-1)$-мерном линейном пространстве глобальных равновесий плазмь, которые не являются трансляционно-инвариантными. Эти точные решения являются либо квазипериодическими, либо периодическими по переменной z.

ДокАЗАТЕльСтво. 1) Предположим, что функция $I(\psi)$ линейна, $I(\psi)=\alpha \psi$, и функция $p(\psi)$ квадратична, $p(\psi)=p_{1}-2 \beta^{2} \psi^{2} / \mu$, где $p_{1}>\left(2 \beta^{2} / \mu\right) \max \left(\psi^{2}(x, y, z)\right)$ и $\alpha$ и $\beta>0$ являются произвольньми постоянньми (константа $p_{1}$ не входит в уравнения равновесия плазмы (1.1)). Отметим, что линейная подстановка для $I(\psi)$ и квадратичная для $p(\psi)$ использовались в статьях [15], [17], [52]-[54]. Однако точные равновесия плазмы, выведенные в наших работах [1]-[3] и представленные в данном обзоре, являются более обшими, чем решения, найденные в [15], [17], [52]-[54]. Ток (2.2) принимает вид

$$
\mathbf{J}=-\frac{\alpha}{\mu} \mathbf{B}+\frac{4 \beta^{2}}{\mu} \psi r \widehat{\mathbf{e}}_{\phi}
$$

Из формул (2.1) и (2.4) следует асимптотика $\mathbf{J} \approx \alpha \mathbf{B} / \mu$ при $r \rightarrow 0$ и $\alpha \neq 0$. Уравнение Грэда-Шафранова с новой переменной $x=2 \beta r^{2}$ принимает вид

$$
x \psi_{x x}+\frac{1}{8 \beta} \psi_{z z}+\frac{1}{8 \beta} \alpha^{2} \psi-\frac{1}{4} x \psi=0 .
$$


Проверка доказывает, что гауссовское распределение

$$
\psi(r, z)=\frac{1}{2 \beta} e^{-\beta r^{2}}=\frac{1}{2 \beta} e^{-x / 2}
$$

является точным решением уравнения (2.5) при $\alpha=0$ и $\beta>0$. Соответствуюшие параметры $\mathbf{B}, \mathbf{J}$ и $р$ имеют вид

$$
\mathbf{B}=e^{-\beta r^{2}} \widehat{\mathbf{e}}_{z}, \quad \mathbf{J}=\frac{2 \beta}{\mu} e^{-\beta r^{2}} r \widehat{\mathbf{e}}_{\phi}, \quad p=p_{1}-\frac{1}{2 \mu} e^{-2 \beta r^{2}} .
$$

Поэтому гауссовское распределение (2.6) определяет $z$-инвариантное глобальное равновесие плазмы.

Подставляя $\psi(x, z)=e^{-\beta r^{2}} u(x, z)$ в уравнение $(2.5)$, мы выводим

$$
x u_{x x}-x u_{x}+\frac{1}{8 \beta}\left(\alpha^{2} u+u_{z z}\right)=0 .
$$

Разделяя переменные посредством подстановки $u(x, z)=P(x) T(z)$, мы получаем

$$
x P^{\prime \prime}-x P^{\prime}+\frac{\alpha^{2}+\lambda}{8 \beta} P=0, \quad T^{\prime \prime}=\lambda T .
$$

Для $\lambda=-\omega^{2}$ мы находим $T(z)=a \cos (\omega z)+b \sin (\omega z)$. Для первого уравнения (2.8) нас интересуют только полиномиальные решения $P(x)$. Такие решения существуют тогда и только тогда, когда $\left(\alpha^{2}-\omega^{2}\right) /(8 \beta)=n$, где $n \geqslant 0$ есть целое число. Отсюда мы получаем конечный спектр допустимых значений $\omega=\omega_{0}, \ldots, \omega_{N}$ :

$$
\omega_{n}=\sqrt{\alpha^{2}-8 \beta n}, \quad n=0,1, \ldots, N, \quad N=\left[\frac{\alpha^{2}}{8 \beta}\right] .
$$

Первое уравнение (2.8) принимает вид $x P^{\prime \prime}-x P^{\prime}+n P=0$. Дифференщируя по $x$, мы получаем $x L^{\prime \prime}+(1-x) L^{\prime}+(n-1) L=0$, где $L(x)=P^{\prime}(x)$. Это уравнение определяет классические полиномы Лагерра $L_{n-1}(x)$ [55]-[57]:

$$
L_{m}(x)=\frac{1}{m !} e^{x} \frac{d^{m}}{d x^{m}}\left(e^{-x} x^{m}\right) .
$$

Поэтому многочлены $P(x)$ являются первообразными функциями полиномов Лагерра. Мы обозначим их $L_{n}^{*}(x)$ :

$$
\begin{gathered}
L_{n}^{*}(x)=\int_{0}^{x} L_{n-1}(t) d t=-\frac{x}{n !} e^{x} \frac{d^{n}}{d x^{n}}\left(e^{-x} x^{n-1}\right), \quad L_{0}^{*}(x)=-1, \\
L_{n}^{*}(x)=x+\sum_{k=1}^{n-1} \frac{(-1)^{k}(n-1) !}{k !(k+1) !(n-k-1) !} x^{k+1} .
\end{gathered}
$$

Отсюда мы получаем точные решения уравнения (2.5):

$$
\psi_{n}(r, z)=\frac{1}{2 \beta} e^{-\beta r^{2}}\left(a_{n} \cos \left(\omega_{n} z\right)+b_{n} \sin \left(\omega_{n} z\right)\right) L_{n}^{*}\left(2 \beta r^{2}\right) .
$$


Для любых постоянных $\alpha$ и $\beta$ формулы (2.9) и (2.11) определяют $N$ точных решений линейного уравнения (2.5). Эти решения удовлетворяют вышеуказанным условиям а) и b). Однако условие с) не выполнено, поскольку магнитная поверхность $\psi_{n}(r, z)=0$ не является ограниченной по переменной $r$, так как она содержит бесконечное число плоскостей $z=z_{k}=z_{0}+2 \pi k / \omega_{n}$.

2) Для получения глобальных решений, удовлетворяюших всем трем условиям а), b) и с), мы полагаем $\alpha^{2}=8 \beta N$. Тогда $\omega_{N}=0$, и точное решение $(2.11)$ принимает вид

$$
\psi_{N}(r)=\frac{a_{N}}{2 \beta} e^{-\beta r^{2}} L_{N}^{*}\left(2 \beta r^{2}\right)
$$

что переходит в гауссовское распределение (2.6) при $N=0$. Соответствующее магнитное поле (2.1), ток (2.4) и давление $p$ имеют вид

$$
\begin{aligned}
\mathbf{B}_{N} & =a_{N} e^{-\beta r^{2}}\left(\left(L_{N}^{*}(x)-2 L_{N}^{*^{\prime}}(x)\right) \widehat{\mathbf{e}}_{z}+\frac{\sqrt{2 N / \beta}}{r} L_{N}^{*}(x) \widehat{\mathbf{e}}_{\phi}\right), \\
\mathbf{J}_{N} & =-\frac{\sqrt{8 \beta N}}{\mu} \mathbf{B}_{N}+\frac{4 \beta^{2}}{\mu} \psi_{N}(r) r \widehat{\mathbf{e}}_{\phi}, \quad p=p_{1}-\frac{a_{N}^{2}}{2 \mu} e^{-2 \beta r^{2}} L_{N}^{* 2}\left(2 \beta r^{2}\right) .
\end{aligned}
$$

Это есть некоторое $z$-инвариантное глобальное равновесие плазмы.

Линейная комбинация функций магнитного потока (2.11) и (2.12) определяет $(2 N-1)$-мерное линейное пространство точных решений уравнения $(2.5)$ :

$$
\begin{aligned}
\psi(r, z)= & \frac{1}{2 \beta} \exp \left(-\beta r^{2}\right)\left(a_{N} L_{N}^{*}\left(2 \beta r^{2}\right)\right. \\
& \left.+\sum_{n=1}^{N-1}\left(a_{n} \cos \left(\omega_{n} z\right)+b_{n} \sin \left(\omega_{n} z\right)\right) L_{n}^{*}\left(2 \beta r^{2}\right)\right),
\end{aligned}
$$

где $\omega_{n}=\sqrt{8 \beta(N-n)}$, коэффициенты $a_{n}, b_{n}$ являются произвольньми и $a_{N} \neq 0$. Давление $p(\psi)=p_{1}-2 \beta^{2} \psi^{2} / \mu$ является квадратичной функцией коэффициентов $a_{n}$, $b_{n}, a_{N}$. Из формул (2.10) следует, что точные решения (2.15) при $r \rightarrow 0$ имеют асимптотический вид

$$
\begin{gathered}
\psi(r, z) \approx C_{N}(z) r^{2} \\
C_{N}(z)=a_{N}+\sum_{n=1}^{N-1}\left(a_{n} \cos \left(\omega_{n} z\right)+b_{n} \sin \left(\omega_{n} z\right)\right) .
\end{gathered}
$$

Поэтому магнитное поле (2.1) и плотность тока (2.4) имеют гладкие асимптотики при $r \rightarrow 0$ :

$$
\mathbf{B} \approx C_{N}^{\prime}(z) r \widehat{\mathbf{e}}_{r}-2 C_{N}(z) \widehat{\mathbf{e}}_{z}+\sqrt{8 \beta N} C_{N}(z) r \widehat{\mathbf{e}}_{\phi}, \quad \mathbf{J} \approx-\frac{8 \beta N}{\mu} \mathbf{B} .
$$

Полоидальные проекции магнитных силовых линий совпадают с линиями уровня $\psi(r, z)=$ const [4], [5]. Из формулы (2.15) следует, что эти кривые приближаются к прямьм линиям $r=$ const при $r \gg 1$, так как ее главньй член имеет вид $-a_{N}\left(-2 \beta r^{2}\right)^{N} \exp \left(-\beta r^{2}\right) /(2 \beta N$ !). Поэтому все магнитные силовые линии и все 
линии тока являются ограниченньпи по радиальной переменной $r$. Таким образом, точные решения $(2.15)$ при $a_{N} \neq 0$ удовлетворяют всем трем условиям a), b), c).

При $N=2$ решения (2.15) являются периодическими функциями от $z$ с периодом $T=\pi / \sqrt{2 \beta}$. При $N \geqslant 3$ решения (2.15) в общем положении являются квазипериодическими функциями от $z$. Действительно, частоты $\omega_{n}=\sqrt{8 \beta(N-n)}$ при $n=N-1$, $N-2, \ldots, 1$ пропорциональны последовательности квадратных корней (1.3), которая, очевидно, содержит рационально независимые числа. Поэтому общие решения (2.15) являются квазипериодическими функциями от $z$, например если $a_{N-1} \neq 0, a_{N-2} \neq 0$.

ЗАмЕчАниЕ 1 . При $\beta \rightarrow 0$ и $r<C<1 / \sqrt{\beta},|z| \ll 1 / \sqrt{8 \beta N}$ функция магнитного потока (2.15) принимает вид $\psi(r, z)=c_{N} r^{2}, C_{N}=a_{1}+\cdots+a_{N}$. Эта функция определяет однородное магнитное поле

$$
\mathbf{B}=-2 C_{N} \widehat{\mathbf{e}}_{z}, \quad \mathbf{J}=0, \quad p=p_{1}
$$

Отсюда мы получаем, что равновесия плазмы (2.15) в пределе $\beta \rightarrow 0$ переходят при $r<C<1 / \sqrt{\beta},|z| \ll 1 / \sqrt{8 \beta N}$ в однородное равновесие (2.17). То же самое справедливо для гауссовского распределения (2.7) и для $z$-инвариантных равновесий плазмы (2.12).

\section{§. Примеры квазипериодических и периодических равновесий плазмы}

I. Первые семь многочленов $L_{n}^{*}(x)(2.10)$ имеют вид

$$
\begin{gathered}
L_{0}^{*}(x)=-1, \quad L_{1}^{*}(x)=x, \quad L_{2}^{*}(x)=-\frac{1}{2} x^{2}+x, \quad L_{3}^{*}(x)=\frac{1}{6} x^{3}-x^{2}+x, \\
L_{4}^{*}(x)=-\frac{1}{24} x^{4}+\frac{1}{2} x^{3}-\frac{3}{2} x^{2}+x, \quad L_{5}^{*}(x)=\frac{1}{120} x^{5}-\frac{1}{6} x^{4}+x^{3}-2 x^{2}+x, \\
L_{6}^{*}(x)=-\frac{1}{720} x^{6}+\frac{1}{24} x^{5}-\frac{5}{12} x^{4}+\frac{5}{3} x^{3}-\frac{5}{2} x^{2}+x .
\end{gathered}
$$

Очевидно, что многочлены $L_{n}^{*}(x)(2.10)$ имеют знакопеременные коэффициенты. Поэтому все их действительные корни неотрицательны.

Используя известное тождество [55], [56] для полиномов Лагерра

$$
L_{n+1}^{\prime}(x)=L_{n}^{\prime}(x)-L_{n}(x)
$$

мы выводим тождество

$$
(n+1) L_{n+1}^{*}(x)=(n-x) L_{n}^{*}(x)+x L_{n}^{*^{\prime}}(x) .
$$

Из этого соотношения по индукции следует, что положительные корни многочленов $L_{n+1}^{*}(x)$ и $L_{n}^{*}(x)$ разделяют друг друга, и поэтому каждый многочлен $L_{n}^{*}(x)$ имеет $n-1$ различных положительных корней. Следовательно, все корни многочленов $L_{n}^{*}(x)$ являются простыми, так же как и для полиномов Лагерра $L_{n}(x)$. Пусть $x_{n}-$ наибольший корень многочлена $L_{n}^{*}(x)$ и $y_{n}$ - его наименњший положительньй корень. 
Мы имеем $x_{2}=y_{2}=2, x_{3}=3+\sqrt{3}, y_{3}=3-\sqrt{3}$. Из указанных вьшше свойств следуют неравенства

$$
\cdots<y_{n}<\cdots<y_{3}<y_{2}=x_{2}<x_{3}<\cdots<x_{n}<\cdots .
$$

Используя рекуррентную формулу [55], [56]

$$
(n+1) L_{n+1}-(2 n+1-x) L_{n}+n L_{n-1}=0
$$

для полиномов Лагерра, мы получаем, что $x_{n}>2 n-3$. Функции $L_{n}^{*}\left(2 r^{2}\right) \exp \left(-r^{2}\right)$, определяюшие точные решения (2.12) и (2.15), быстро убывают при $r \rightarrow \infty$, см. графики на рис. 1.

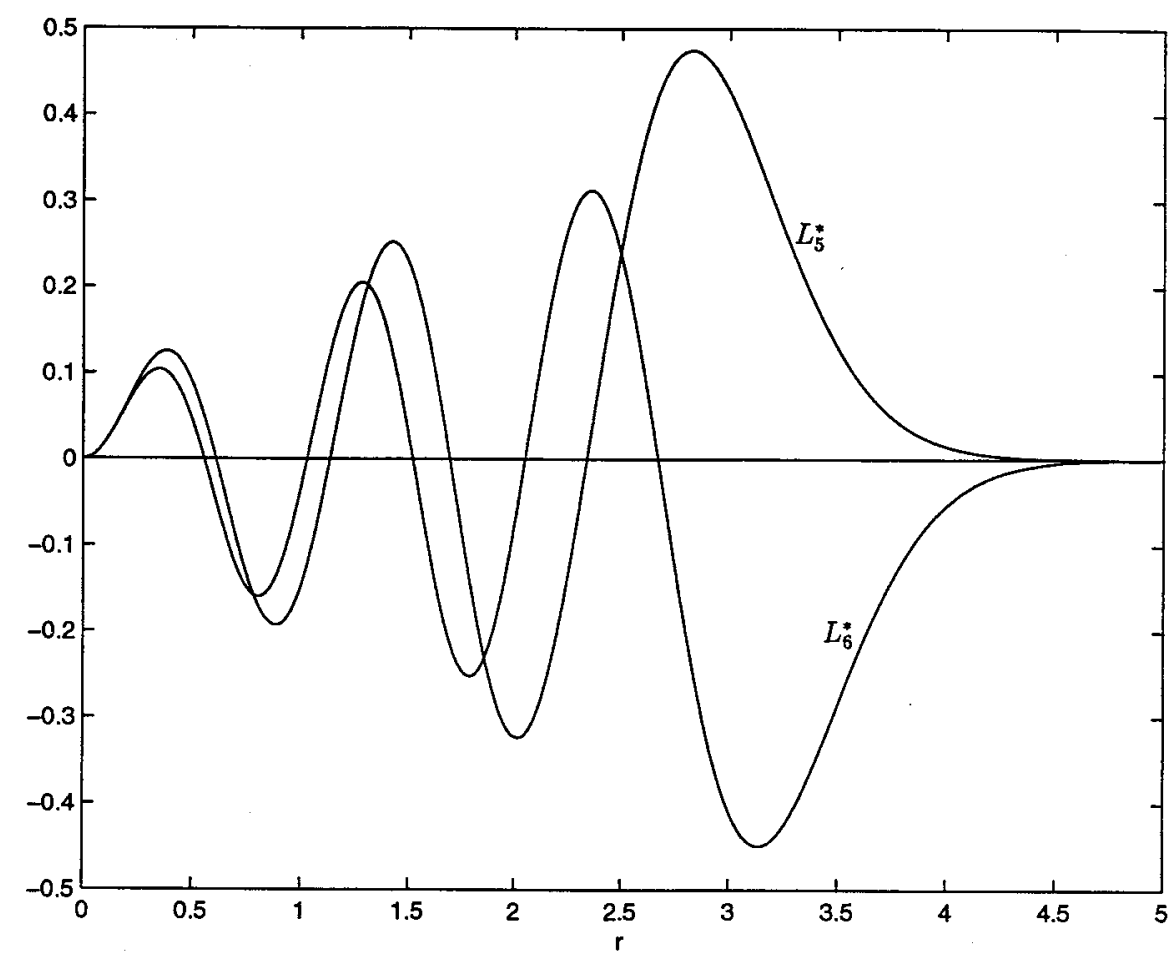

Рис. 1. Графики функций $L_{5}\left(2 r^{2}\right) \exp \left(-r^{2}\right)$ и $L_{6}\left(2 r^{2}\right) \exp \left(-r^{2}\right)$

II. ПРИМЕР 1. МоДЕЛЬ АСТРОФИЗИЧЕСКИХ СтРУЙ. Рис. 2 показывает распределение квазипериодических кривых уровня $\psi(r, z)=$ const для следующего конкретного точного решения $(2.15)$ для $N=3, \beta=0.1, \alpha^{2}=24 \beta$ :

$$
\begin{aligned}
\psi(r, z)= & \frac{1}{2 \beta} \exp \left(-\beta r^{2}\right)\left(L_{3}^{*}\left(2 \beta r^{2}\right)+\frac{1}{20} \sin (2 \sqrt{2 \beta} z) L_{2}^{*}\left(2 \beta r^{2}\right)\right. \\
& \left.+\frac{1}{20} \sin (4 \sqrt{\beta}(z-1)) L_{1}^{*}\left(2 \beta r^{2}\right)\right) .
\end{aligned}
$$


Линии уровня $\psi(r, z)=$ const совпадают с полоидальньми проекциями магнитных силовых линий и линий тока. Эти кривые являются квазипериодическими по переменной $z$, поскольку частоты $2 \sqrt{2 \beta}$ и $4 \sqrt{\beta}$ в $(3.3)$ рационально независимы. При $r>9$ эти кривые практически параллельны оси $z$. Следовательно, характерньй масштаб $l$ (по переменной $r$ ) возмушений $z$-инвариантного равновесия в формуле (3.3) удовлетворяет неравенству $l<9$. Врашая кривые на рис. 2 вокруг оси $z$, мы получаем магнитные поверхности, составляющие непрерывные семейства цилиндров и вложенных торов. Наиболее внутренние торы являются круговыми магнитными осями. Распределение этих инвариантных тороидальных областей и их магнитных осей является квазипериодическим по переменной $z$. Магнитное поле имеет довольно сложную топологию: замкнутые магнитные силовые линии плотны в каждой тороидальной области и в общем случае зацеплены друг с другом.

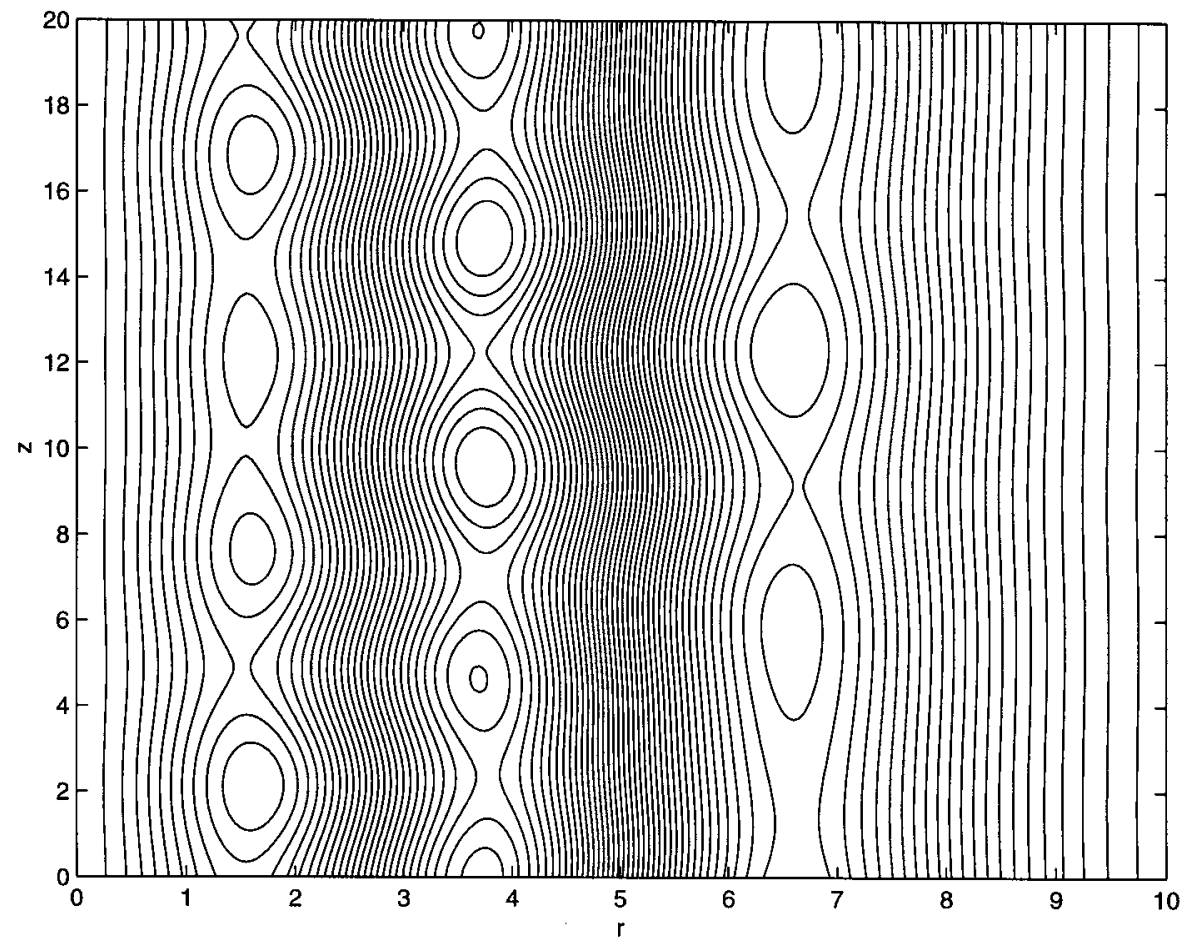

Рис. 2. Квазипериодические магнитные силовые линии для модели астрофизических струй

Рис. 3 представляет плотность магнитной энергии $B^{2}(x, y, z) /(2 \mu)$ для равновесия плазмы (3.3) для $y=0, z=0.5$ и $\beta=0.1, \mu=0.5$. Очевидно, что $B^{2}(r, z) \neq 0$ всюду и что $B^{2}(r, z) \rightarrow 0$ при $r \rightarrow \infty$. Магнитная энергия сконцентрирована около оси симметрии. Это свойство означает, что указанное вьше точное решение моделирует некоторую астрофизическую струю в сопутствуюшей системе отсчета.

Рис. 4 показывает давление плазмы $p=p_{1}-2 \beta^{2} \psi^{2}(r, z) / \mu$ для равновесия (3.3), где мы полагаем $p_{1}=8.2, \beta=0.1, z=0.5, \mu=0.5$. Очевидно, что $p \approx p_{1}$ при 


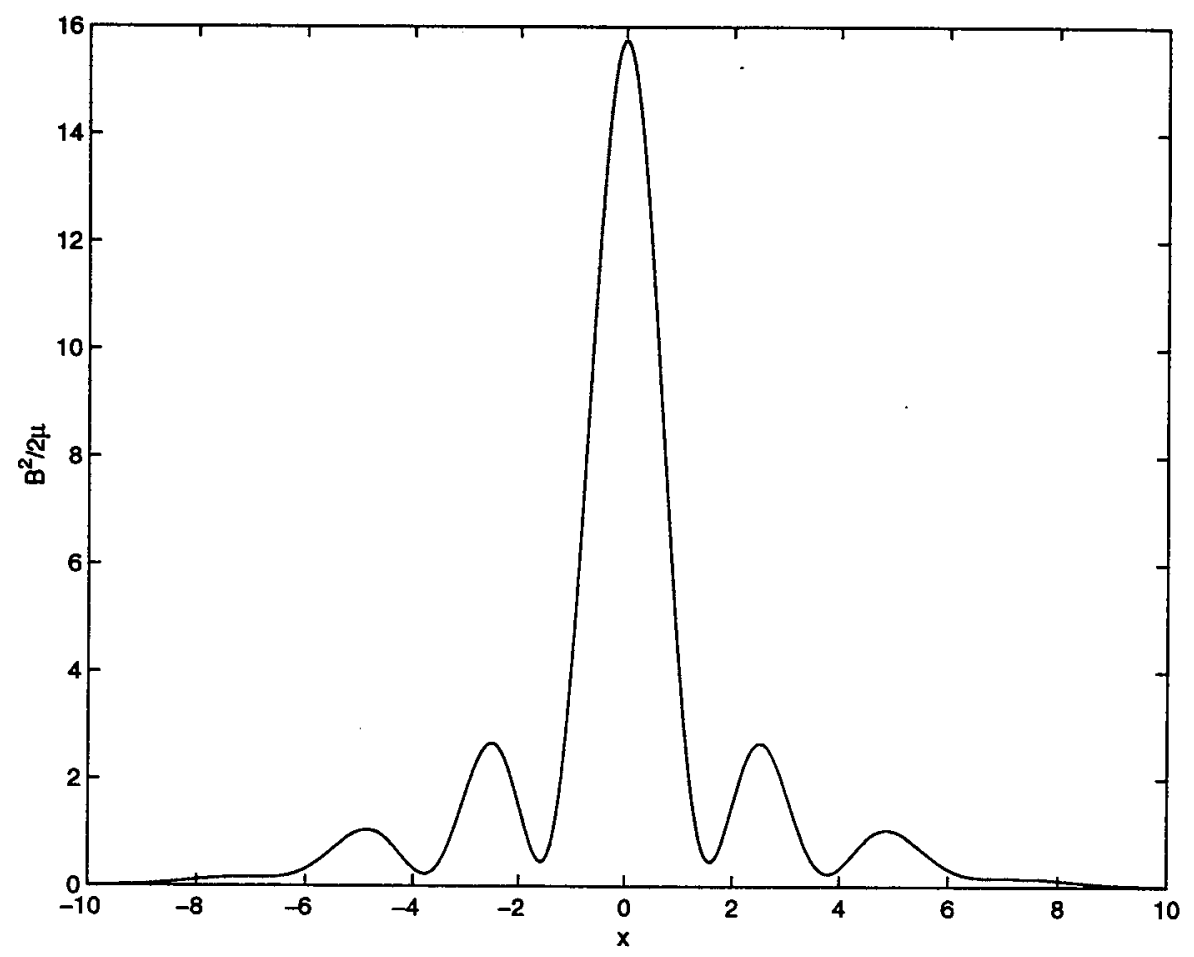

Рис. 3. Плотность магнитной энергии $B^{2}(x, 0,0.5) /(2 \mu)$ для модели астрофизических струй

$r>10$ и при $r<0.5$. Константа $p_{1}$ является средним давлением в соответствуюшем истекающем потоке.

III. Рассмотрим подмножество решений (2.15), где ненулевые коэффициенты $a_{n} \neq 0$ соответствуют $n=n(k)=N-k^{2}$. Пусть $L=[\sqrt{N-1}]$. Тогда функция $(2.15)$ принимает вид

$$
\begin{aligned}
\psi(r, z)= & \frac{1}{2 \beta} \exp \left(-\beta r^{2}\right)\left(a_{N} L_{N}^{*}\left(2 \beta r^{2}\right)\right. \\
& \left.+\sum_{k=1}^{L}\left(a_{n(k)} \cos (\sqrt{8 \beta} k z)+b_{n(k)} \sin (\sqrt{8 \beta} k z)\right) L_{n(k)}^{*}\left(2 \beta r^{2}\right)\right) .
\end{aligned}
$$

Эта функция имеет вид ряда Фурье с произвольными коэффициентами $a_{n(j)}, a_{N}$. Поэтому точные решения (3.4) для любого $r=r_{0}$ приближают любую гладкую функцию $\bar{\psi}\left(r_{0}, z\right)$, которая является $(\pi / \sqrt{2 \beta})$-периодической по переменной $z$. Таким образом, даже подмножество $z$-периодических точных решений (3.4) представляет довольно общие глобальные осесимметричные равновесия плазмы.

ПримеР 2. Простейшее $z$-периодическое равновесие плазмы имеет функцию магнитного потока

$$
\psi(r, z)=\frac{1}{2 \beta} e^{-\beta r^{2}}\left(L_{2}^{*}\left(2 \beta r^{2}\right)+a_{1} \sin (\sqrt{8 \beta} z) L_{1}^{*}\left(2 \beta r^{2}\right)\right) .
$$




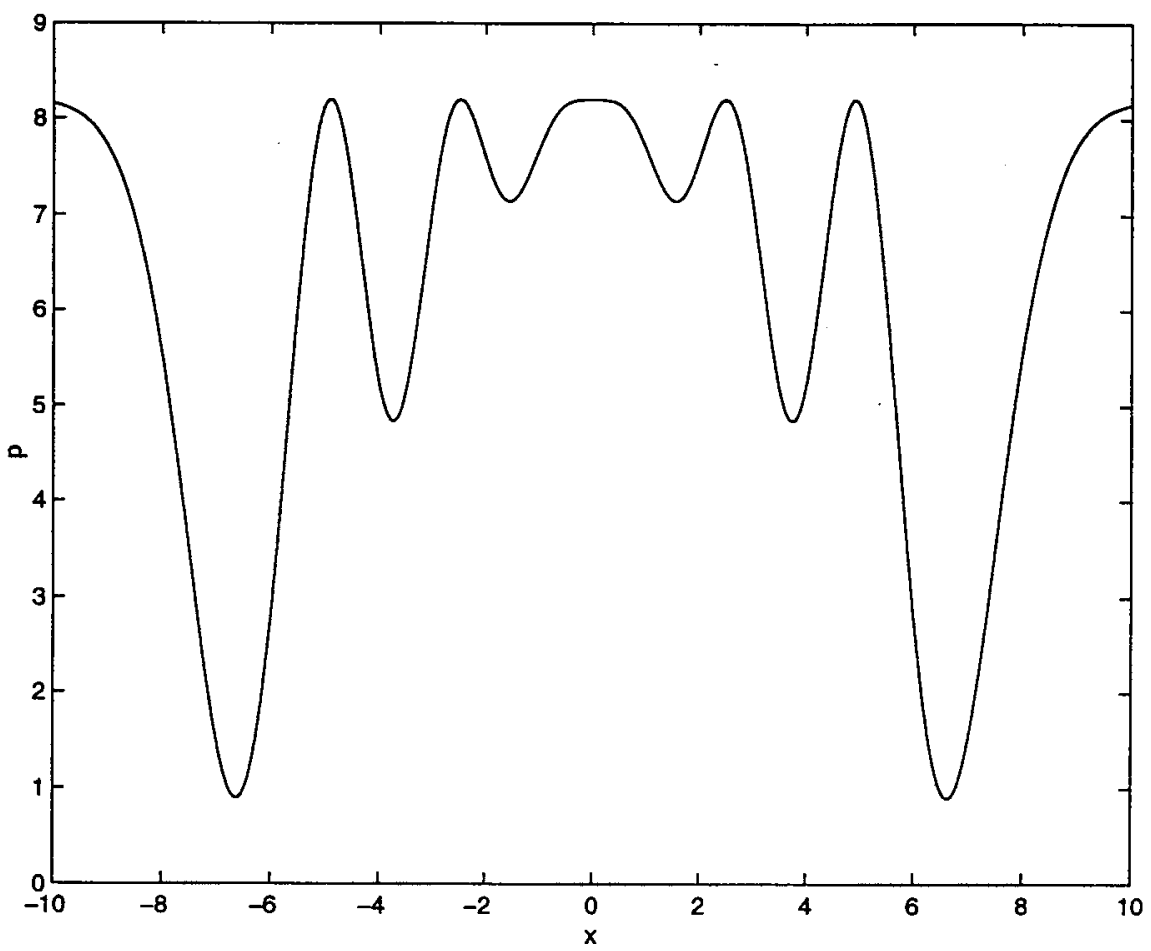

Рис. 4. Давление плазмы $p(x, 0,0.5)$ для модели астрофизических струй

Соответствующие линии уровня $\psi(r, z)=$ const при $\beta=0.1, a_{1}=0.1$ показаны на рис. 5 , откуда с очевидностью следует, что характерный масштаб $l$ (по переменной $r$ ) возмущений $z$-инвариантного равновесия в (3.5) удовлетворяет неравенству $l<9$.

\section{$\S$ 4. Квазипериодичность глобальньх равновесий плазмы}

I. Зависимость функции магнитного потока (2.15) от переменой $z$ определяется $N-1$ частотами $\omega_{n}=\sqrt{8 \beta(N-n)}$, принадлежашими сегменту $I_{N}$ между минимальной $\omega_{N-1}=\sqrt{8 \beta}$ и максимальной $\omega_{1}=\sqrt{8 \beta(N-1)}$ частотами. Распределение частот $\omega_{n}$ на сегменте $I_{N}$ не является однородным. Внутри каждого из равных интервалов $(k \sqrt{8 \beta},(k+1) \sqrt{8 \beta}) \subset I_{N}$ имеется $2 k$ частот $\omega_{N-k^{2}}, \ldots, \omega_{N-(k+1)^{2}}$. Поэтому функция плотности частот $D(\omega)$ имеет вид $D(\omega) \approx \omega /(4 \beta)$. Это свойство показывает, что решения (2.15) сильно отличаются от рядов Фурье, для которых все частоты являются целыми кратными базисной частоты.

II. Аналитическая сложность $z$-квазипериодической функции $\psi(r, z)(2.15)$ определяется точным числом рационально независимых частот $\omega_{n}$ или числом рационально независимых квадратных корней в последовательности (1.3). Мы обозначаем это число $d(N)$. Функция $d(N)$ довольно нетривиальна. Например, для $N=19$ последовательность (1.3) принимает вид

$$
1, \sqrt{2}, \sqrt{3}, 2, \sqrt{5}, \sqrt{6}, \sqrt{7}, 2 \sqrt{2}, 3, \sqrt{10}, \sqrt{11}, 2 \sqrt{3}, \sqrt{13}, \sqrt{14}, \sqrt{15}, 4, \sqrt{17}, 3 \sqrt{2} \text {. }
$$




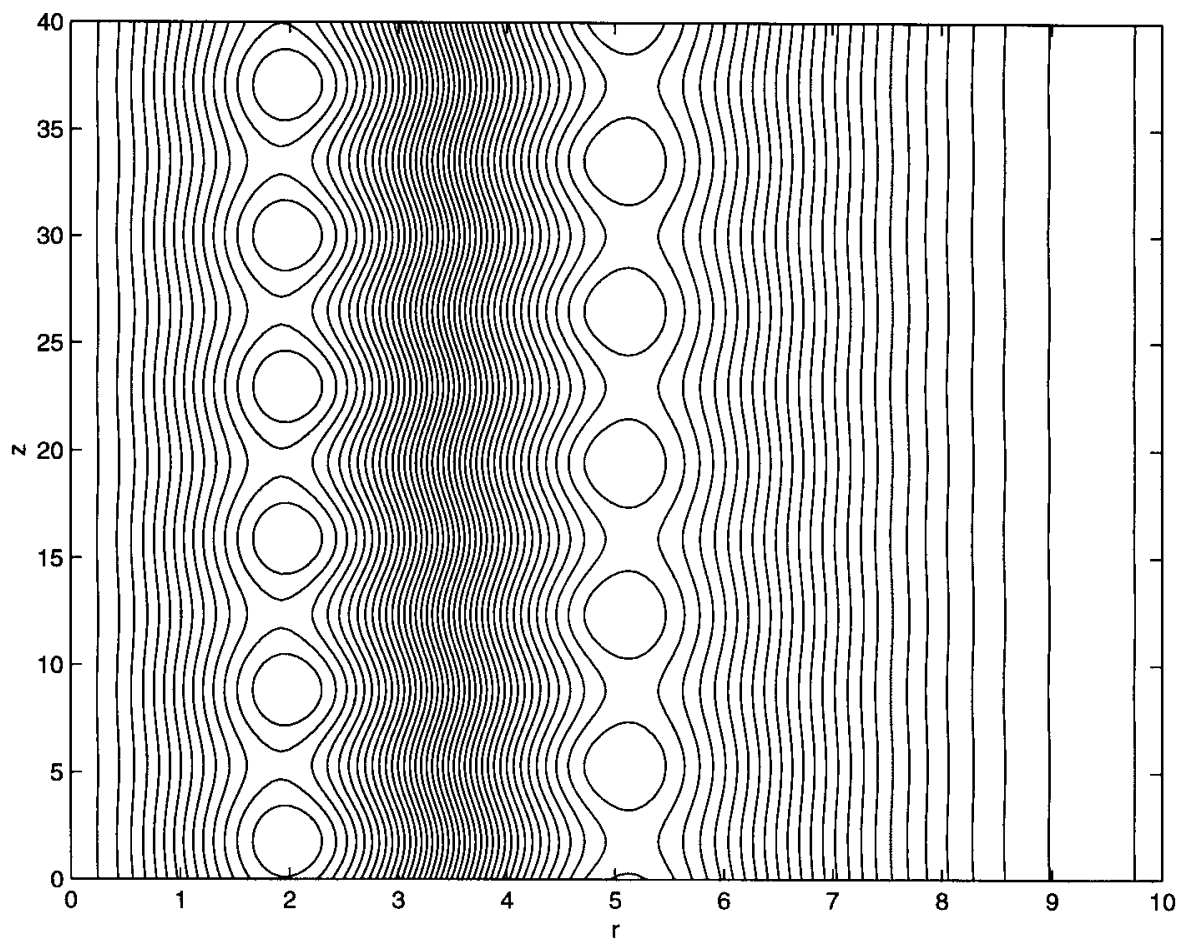

Рис. 5. Периодические по $z$ полоидальные магнитные силовые линии

Поэтому функция $d(N)$ при $N=2,3, \ldots, 19$ имеет следующие последовательные значения

$$
d(N)=1,2,3,3,4,5,6,6,6,7,8,8,9,10,11,11,12,12 .
$$

Следовательно, число рационально независимых частот $\sqrt{8 \beta(N-n)}$ для $N=19$ равно 12.

Очевидно, что если $m_{1}=p_{1}^{k_{1}} \cdots p_{n}^{k_{n}}$, то квадратный корень $\sqrt{m_{1}}$ рационально зависим с $\sqrt{m_{0}}$, где $m_{0}=p_{i_{1}} \cdots p_{i_{k}}$. Поэтому базис рационально независимых корней в (1.3) не больше чем множество корней $\sqrt{p_{1} \cdots p_{n}}$, где $p_{j}$ - различные простые числа. Квадратные корни $\sqrt{p_{1} \cdots p_{n}}$ являются рационально независимыми. Действительно, предположим, что $p_{n}$ есть первое простое число такое, что существует некоторая рациональная зависимость между действительными числами $\sqrt{p_{j_{1}} \cdots p_{j_{m}}}$, где $p_{j} \leqslant p_{n}$ являются несовпадающими простыми числами. Тогда справедливо некоторое уравнение

$$
\sqrt{p_{n}}\left(\sum_{\alpha} c_{\alpha} \sqrt{p_{\alpha(1)} \cdots p_{\alpha(k)}}\right)=\sum_{\beta} c_{\beta} \sqrt{p_{\beta(1)} \cdots p_{\beta(m)}},
$$

где $c_{\alpha}, c_{\beta}$ являются рациональными числами и $p_{\alpha(j)}, p_{\beta(k)}<p_{n}$. Уравнение (4.1) означает, что $\sqrt{p_{n}}$ принадлежит алгебраическому расширению поля рациональных чисел $\mathbb{Q}$, порожденному квадратными корнями $\sqrt{2}, \sqrt{3}, \ldots, \sqrt{p_{n-1}}$. Однако классическая теорема о квадратичных расширениях [58] утверждает, что это не верно. Полученное противоречие доказьвает, что все числа $\sqrt{p_{1} \cdots p_{n}}$ рационально независимы. Этот результат следует также из более общей теоремы Безиковича [59]. 
Таким образом, функция $d(N)$ равна числу составных чисел $p_{1} \cdots p_{n}<N$, не содержащих квадратов. Асимптотика этой функции при $N \rightarrow \infty$ известна из теории чисел:

$$
d(N) \approx \frac{1}{\zeta(2)} N=\frac{6}{\pi^{2}} N
$$

см. [60]. Отсюда мы получаем, что около трех пятых всех частот $\omega_{n}$ в (1.3) являются рационально независимыми.

\section{$\S 5$. Глобальные равновесия плазмы в полупространстве}

Применим развитые методы к проблеме равновесия плазмы в полупространстве $z \geqslant 0$. Эта проблема хорошо известна в теории солнечных протуберанцев [11]. Полагая в уравнениях $(2.8)$

$$
\lambda=\kappa_{n}^{2}, \quad \kappa_{n}=\sqrt{8 \beta n-\alpha^{2}}, \quad n=N, N+1, \ldots, \quad N \geqslant \frac{\alpha^{2}}{8 \beta},
$$

мы находим при $\alpha^{2} \leqslant 8 \beta N$ точные решения $P_{n}(x)=L_{n}^{*}(x), T_{n}(z)=\exp \left(-\kappa_{n} z\right)$. Отсюда следует, что линейное уравнение (2.5) имеет точные решения

$$
\psi(r, z)=e^{-\beta r^{2}} \sum_{n=N}^{N+m} a_{n} \exp \left(-\sqrt{8 \beta n-\alpha^{2}} z\right) L_{n}^{*}\left(2 \beta r^{2}\right),
$$

где $\alpha, \beta>0$ и $a_{n}$ являются произвольными постоянными и $m \geqslant 0, N \geqslant 0$ - произвольные целые числа. Решения (5.1) определены в полупространстве $z \geqslant 0$ и быстро стремятся к нулю при $r \rightarrow \infty$ и при $z \rightarrow \infty$, если $\alpha^{2}<8 \beta N$. Давление плазмы $p$ имеет вид $p(\psi)=p_{1}-2 \beta^{2} \psi^{2} / \mu$, где $p_{1}>\left(2 \beta^{2} / \mu\right) \max \left(\psi^{2}(r, z)\right), z \geqslant 0$. Соответствуюшие магнитное поле (2.1), ток (2.4) и давление $p$ не имеют сингулярностей в полупространстве $z \geqslant 0$. Поэтому точные решения (5.1) удовлетворяют физическим условиям а) и b). Они удовлетворяют также условию с), если $\operatorname{sign} a_{n}=(-1)^{n}$, поскольку тогда все слагаемые в сумме (5.1) имеют одинаковый знак при $r \gg 1$, что имеет следствием ограниченность по $r$ всех магнитных поверхностей. Поэтому полученные решения определяют глобальные равновесия плазмы в полупространстве $z \geqslant 0$.

Наиболее важное физическое свойство точных решений (5.1) при $\alpha^{2}<8 \beta N$ состоит в том, что их полная магнитная энергия

$$
E_{m}=\int \frac{B^{2}}{2 \mu} d V
$$

в полупространстве $z \geqslant 0$ является конечной. Эти глобальные равновесия плазмы моделируют солнечные протуберанщы; асимптотическое значение давления $p_{1}$ является средним давлением плазмы в солнечной короне.

ПрИМеР 3. МоДЕЛЬ СОЛнЕчныХ ПРОтУБЕРАнцев. Рис. 6 представляет полоидальные магнитные силовые линии для точного решения вида (5.1):

$$
\psi(r, z)=e^{-\beta r^{2}}\left(e^{-4 \sqrt{\beta} z} L_{2}^{*}\left(2 \beta r^{2}\right)-0.5 e^{-2 \sqrt{6 \beta} z} L_{3}^{*}\left(2 \beta r^{2}\right)\right)
$$




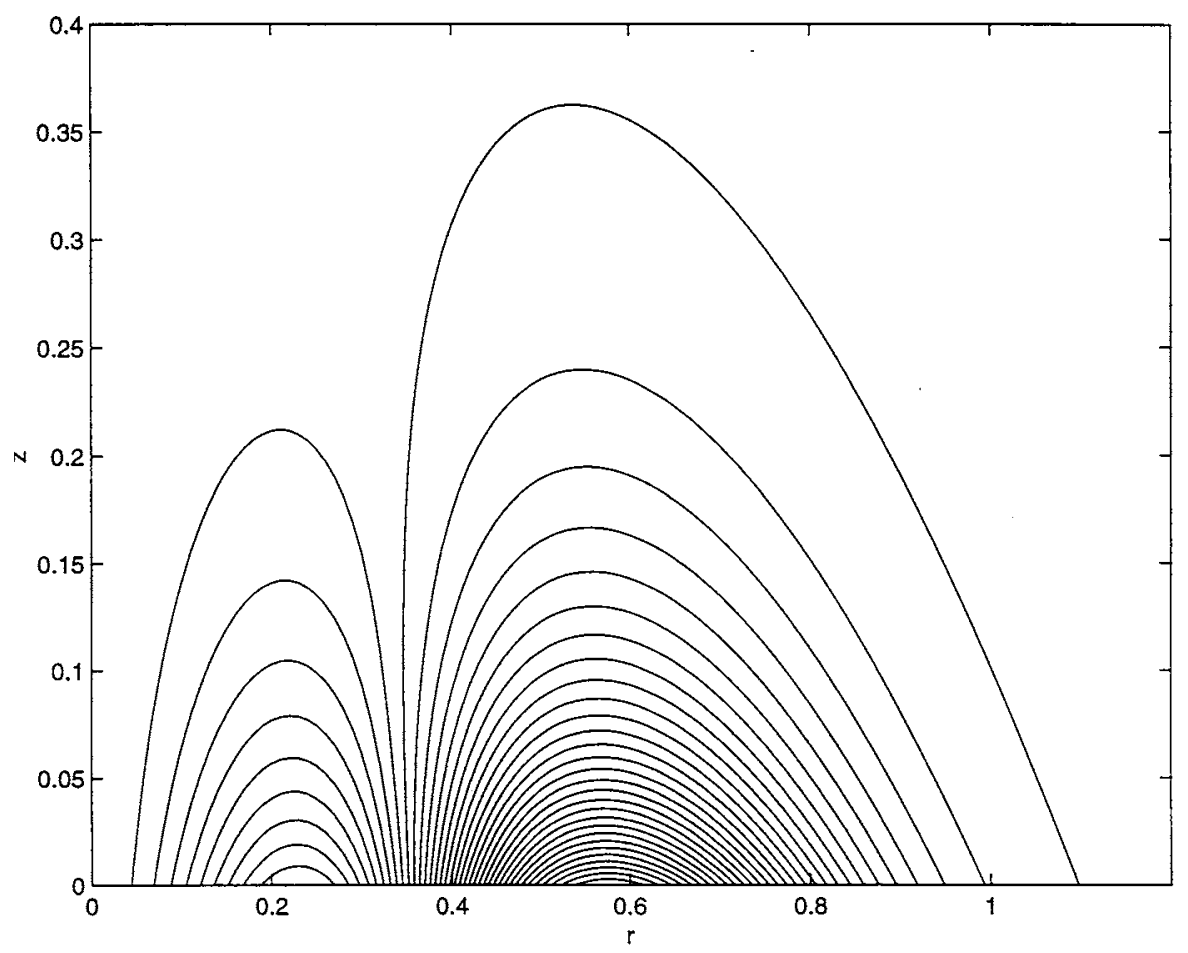

Рис. 6. Магнитные силовые линии для модели солнечных протуберанцев

при $\alpha=0$ и $\beta=10$. Соответствующее магнитное поле $(2.1)$ и ток $(2.4)$ имеют вид

$$
\mathbf{B}=\frac{\psi_{z}}{r} \widehat{\mathbf{e}}_{r}-\frac{\psi_{r}}{r} \widehat{\mathbf{e}}_{z}, \quad \mathbf{J}=\frac{4 \beta^{2} r \psi}{\mu} \widehat{\mathbf{e}}_{\phi}
$$

Магнитное поле В (5.3) является чисто полоидальным, ток $\mathbf{J}$ (5.3) является чисто круговым. Все магнитные силовые линии начинаются и заканчиваются при $z=0$ (гранища солнечной фотосферы). Трехмерная структура магнитного поля В получается из рис. 6 врашением вокруг оси $z$.

При $\alpha^{2}=8 \beta N$ функция магнитного потока (5.1) принимает вид

$$
\psi(r, z)=e^{-\beta r^{2}}\left(a_{N} L_{N}^{*}\left(2 \beta r^{2}\right)+\sum_{n=N+1}^{N+m} a_{n} \exp (-\sqrt{8 \beta(n-N)} z) L_{n}^{*}\left(2 \beta r^{2}\right)\right)
$$

Эти точные решения образуют $(m+1)$-мерное линейное пространство возмушений цилиндрически симметричного равновесия плазмы (2.12) в полупространстве $z \geqslant 0$. При $z \rightarrow \infty$ эти решения стремятся к равновесию $(2.12)$.

\section{§6. Контрпримеры к теореме Паркера}

I. Выведенные z-инвариантные равновесия плазмы (2.12) имеют следующее важное свойство. Соответствующее магнитное поле $\mathbf{B}_{N}(2.13)$ и электрический ток $\mathbf{J}_{N}$ 
(2.14) отличны от нуля во всем евклидовом пространстве $\mathbb{R}^{3}$. Действительно, если, например, компонента $(2.13) B_{N_{\phi}}\left(x_{1}\right)=0$, то $L_{N}^{*}\left(x_{1}\right)=0$; отсюда $L_{N}^{*}\left(x_{1}\right) \neq 0$, поскольку все корни многочлена $L_{N}^{*}(x)$ являются простыми. Поэтому компонента $B_{N_{z}}\left(x_{1}\right) \neq 0$. Следовательно, в любой области $0 \leqslant r \leqslant R$ мы имеем

$$
\left|\mathbf{B}_{N}\right|>B\left|a_{N}\right|>0, \quad\left|\mathbf{J}_{N}\right|>J\left|a_{N}\right|>0 .
$$

Для точных решений (2.15) вариации магнитного поля $\mathbf{B}-\mathbf{B}_{N}$ и вариации тока $\mathbf{J}-\mathbf{J}_{N}$ определяются функцией магнитного потока

$$
\psi-\psi_{N}=\frac{1}{2 \beta} e^{-\beta r^{2}} \sum_{n=1}^{N-1}\left(a_{n} \cos \left(\omega_{n} z\right)+b_{n} \sin \left(\omega_{n} z\right)\right) L_{n}^{*}\left(2 \beta r^{2}\right) .
$$

Используя формулы $(2.1),(2.15)$ и (6.2), мы получаем (то же самое для $\mathbf{J})$

$$
\frac{\left|\mathbf{B}-\mathbf{B}_{N}\right|}{\left|\mathbf{B}_{N}\right|}<\frac{\left|\mathbf{B}-\mathbf{B}_{N}\right|}{B\left|a_{N}\right|}<A_{N} \frac{C(R)}{B}, \quad A_{N}=\frac{1}{\left|a_{N}\right|} \sum_{n=1}^{N-1}\left(\left|a_{n}\right|+\left|b_{n}\right|\right)
$$

в области $0 \leqslant r \leqslant R$, где $C(R)$ - некоторая гладкая функция. Формулы (6.1) и (6.3) означают, что неравенства

$$
\frac{\left|\mathbf{B}-\mathbf{B}_{N}\right|}{\left|\mathbf{B}_{N}\right|} \ll 1, \quad \frac{\left|\mathbf{J}-\mathbf{J}_{N}\right|}{\left|\mathbf{J}_{N}\right|} \ll 1
$$

справедливы в области $0 \leqslant r \leqslant R$ при условии, что $A_{N} \ll 1$.

При $r \rightarrow \infty$ функция магнитного потока $\psi_{N}(2.12)$ и ее вариации $\psi-\psi_{N}(6.2)$ имеют следуюшие асимптотики:

$$
\begin{gathered}
\psi_{N}(r, z) \approx-\frac{a_{N}\left(-2 \beta r^{2}\right)^{N}}{2 \beta N !} e^{-\beta r^{2}} \\
\psi-\psi_{N} \approx-\frac{\left(-2 \beta r^{2}\right)^{N}}{2 \beta N !} e^{-\beta r^{2}} \sum_{n=1}^{N-n} \frac{N !}{n !\left(-2 \beta r^{2}\right)^{N-n}}\left(a_{n} \cos \left(\omega_{n} z\right)+b_{n} \sin \left(\omega_{n} z\right)\right) .
\end{gathered}
$$

Отсюда мы получаем

$$
\frac{\psi-\psi_{N}}{\psi_{N}} \rightarrow 0 \text { при } r \rightarrow \infty .
$$

Вследствие (6.6) отношения (6.4) стремятся к нулю при $r \rightarrow \infty$. Поэтому неравенства (6.4) справедливы также при $r>R$ и, следовательно, всюду в евклидовом пространстве $\mathbb{R}^{3}$ при условии, что $A_{N} \ll 1$.

Оценим характерньй масштаб $l$ (по переменной $r$ ) возмушений, представленных суммой в формуле (2.15). Как показано в $\S 3$, каждый многочлен $L_{n}^{*}(x)$ имеет $n-1$ 
различных положительных корней и $\left|a_{n}\right|=1 / n$ ! для его старшего коэффициента. Отсюда мы находим $\left|L_{n}^{*}(x)\right|<x^{n} / n$ !. Если $x>N$, то мы имеем $x^{n} / n$ ! $<x^{N-1} /(N-1)$ ! для всех $n<N-1$. Следовательно, при $x>N$ мы получаем

$$
\left|L_{1}^{*}(x)\right|+\cdots+\left|L_{N-1}^{*}(x)\right|<\frac{x^{N-1}}{(N-2) !} .
$$

Для функции магнитного потока $\psi(2.15)$ мы имеем

$$
\left|\psi-\psi_{N}\right|<\frac{1}{2 \beta}\left|a_{N}\right| A_{N} e^{-\beta r^{2}} \frac{x^{N-1}}{(N-2) !}
$$

Для $x>x_{N}$ мы имеем $L_{N}^{*}(x)>\left(x-x_{N}\right)^{N} / N$ !, где $x_{N}$ является наибольшим корнем многочлена $L_{N}^{*}(x)$. Отсюда

$$
\left|\psi_{N}\right|>\frac{1}{2 \beta}\left|a_{N}\right| e^{-\beta r^{2}} \frac{\left(x-x_{N}\right)^{N}}{N !}
$$

Из формул (6.7) и (6.8) следует

$$
\frac{\left|\psi-\psi_{N}\right|}{\left|\psi_{N}\right|}<A_{N} \frac{N(N-1) x^{N-1}}{\left(x-x_{N}\right)^{N}}<A_{N} \frac{N^{2}}{x\left(1-x_{N} / x\right)^{N}} .
$$

Отсюда мы получаем при $x>N^{2} x_{N}$ :

$$
\frac{\left|\psi-\psi_{N}\right|}{\left|\psi_{N}\right|}<A_{N}
$$

Такие же неравенства справедливы для магнитного поля и электрического тока. Таким образом, при $A_{N} \ll 1$ возмущения (2.15) могут быть значительны только при $x<N^{2} x_{N}$. Подставляя $x=2 \beta l^{2}$, мы получаем для характерной длины $l$ (по переменной $r$ ) возмущений (2.15):

$$
l \leqslant N \sqrt{\frac{x_{N}}{2 \beta}}
$$

Например, для равновесия (3.3) мы имеем $N=3, x_{3}=3+\sqrt{3}, \beta=0.1, A_{3}=0.1$. Отсюда характерный масштаб возмушений $l \leqslant 3 \sqrt{x_{3} /(2 \beta)} \approx 14.59$. Это следует также из рис. 2.

Неравенства (6.4) означают, что равновесия плазмы (2.15) при $A_{N} \ll 1$ являются мальми возмущениями $z$-инвариантного равновесия (2.12) во всем евклидовом пространстве $\mathbb{R}^{3}$. Отсюда мы получаем, что условие Паркера о том, что "локальное возмущение поля является малым по сравнению с полным полем" [23; с. 361], выполнено всюду. Условие Паркера о том, что длина трубки тока $L$ является "большой по сравнению с характерным трансверсальным маситабом $l$ вариации поля" [23; с. 362], выполнено, поскольку $l \leqslant N \sqrt{x_{N} /(2 \beta)}$ и длина трубки тока $L$ может 
быть выбрана произвольно большой для $z$-инвариантного равновесия (2.12). Отсюда $L \gg l$. Условие Паркера о том, что “магнитное поле является аналитическим в его отклонении є от инвариантного поля $B_{i}(x, y)$ " [23; с. 378], вьполнено, так как точные решения (2.15) являются линейными функциями малых параметров $a_{1}, b_{1}, \ldots, a_{N-1}, b_{N-1}$. Все возмущения (2.15) не являются $z$-инвариантньпи. Поэтому равновесия плазмы (2.15) представляют собой контрпримеры к теореме Паркеpa.

Равновесия плазмы (5.4) являются возмущениями z-инвариантных равновесий (2.12) в полупространстве $z \geqslant 0$. Соответствуюшие неравенства (6.4) справедливы при

$$
A_{N}=\frac{1}{\left|a_{N}\right|}\left(\left|a_{N+1}\right|+\cdots+\left|a_{N+m}\right|\right) \ll 1 .
$$

Оценка $l \leqslant N \sqrt{x_{N} /(2 \beta)}$ для характерного масштаба $l$ возмушений (5.4) справедлива при $A_{N} \ll 1$. Условие Паркера об аналитичности по малому параметру $\varepsilon$ очевидно удовлетворено. Все возмушения (5.4) не являются z-инвариантными. Поэтому точные решения (5.4) в полупространстве $z \geqslant 0$ являются контрпримерами к теореме Паркера.

II. ОДИН ИЗ ИСТОЧНИКОВ РАСХОЖДЕНИЯ С РЕЗУЛЬТАТАМИ ПАРКЕРА. В СВоей книге [23] Паркер пишет (с. 369): “Мы предположсим для удобства, что хотя $B_{z}(x, y)$ может иироко изменяться, оно не обращается в нуль и не меняет знак" и приходит к утверждению:

“Результат может бъть написан в виде

$$
\frac{\partial}{\partial x} \frac{1}{B_{z}^{2}} \frac{\partial \Psi}{\partial x}+\frac{\partial}{\partial y} \frac{1}{B_{z}^{2}} \frac{\partial \Psi}{\partial y}+\frac{\partial}{\partial z} \frac{1}{B_{z}^{2}} \frac{\partial \Psi}{\partial z}=0 .
$$

Эта форма является полностью эллиптической. В бесконечном пространстве ее единственными ограниченными решениями являются постоянные, $\Psi=C "$.

Доказательство теоремы Паркера на с. 369-370 [23] и доказательство ее обобщения на магнитную гидродинамику [41; с. 837, уравн. (62)] основаны на этом утверждении. Мы представим контрпример также и к этому утверждению. Выберем функцию $B_{z}(x, y)$ в виде

$$
B_{z}(x, y)=F(h(x, y)), \quad \frac{\partial^{2} h}{\partial x^{2}}+\frac{\partial^{2} h}{\partial y^{2}}=\Delta h=0
$$

где $h(x, y)$ есть произвольная гармоническая функция и $F(u)$ есть произвольная гладкая функция, которая является положительной при всех $u$ и имеет ограниченный интеграл

$$
\int_{-\infty}^{+\infty} F^{2}(u) d u<C_{1}
$$


Например, $F(u)$ может быть гауссовой функцией $e^{-\beta u^{2}}$, использованной выше, и $h(x, y)$ может быть $a x+b y+c x y+d\left(x^{2}-y^{2}\right)$. Функция $B_{z}(x, y)$ (6.11) является положительной при всех $x, y$. Мы определяем функцию $\Psi(x, y, z)$ формулой

$$
\Psi(x, y, z)=\int_{-\infty}^{h(x, y)} F^{2}(u) d u .
$$

Очевидно, что

$$
\frac{\partial}{\partial x} \frac{1}{B_{z}^{2}} \frac{\partial \Psi}{\partial x}=\frac{\partial^{2} h}{\partial x^{2}}, \quad \frac{\partial}{\partial y} \frac{1}{B_{z}^{2}} \frac{\partial \Psi}{\partial y}=\frac{\partial^{2} h}{\partial y^{2}}, \quad \frac{\partial}{\partial z} \frac{1}{B_{z}^{2}} \frac{\partial \Psi}{\partial z}=0 .
$$

Отсюда вследствие $\Delta h=0$ получаем, что функция $\Psi(x, y, z)$ удовлетворяет вышеприведенному эллиптическому уравнению. Она очевидно ограничена, $0<\Psi(x, y, z)<C_{1}$, и не постоянна.

\section{Часть II. Равновесия плазмы с винтовой симметрией}

\section{§ 7. Точные глобальные равновесия плазмы с винтовой симметрией}

Равновесия плазмы с винтовой симметрией были впервые введены Джонсоном, Фриманом, Калсрудом и Оберманом в [38]. В цилиндрических координатах $r, z, \phi$ эти решения зависят от двух переменных $u=z-\gamma \phi$ и $r$ и поэтому инвариантны по отношению к винтовым преобразованиям $z \rightarrow z+\gamma \phi_{0}, \phi \rightarrow \phi+\phi_{0}$. Магнитное поле $\mathbf{B}$, ток $\mathbf{J}$ и давление $p$ являются $2 \pi \gamma$-периодическими функциями переменной $u$ и, следовательно, $z$.

Магнитное поле $\mathbf{B}$ с винтовой симметрией имеет вид

$$
\mathbf{B}=\frac{\psi_{u}}{r} \widehat{\mathbf{e}}_{r}-\frac{\psi_{r}}{r} \widehat{\mathbf{e}}_{u}+\frac{f}{r} \widehat{\mathbf{e}}_{\phi},
$$

где $\psi(u, r)$ и $f(u, r)$ - некоторые гладкие функции, $\psi_{x}=\partial \psi / \partial x$ и $\widehat{\mathbf{e}}_{r}, \widehat{\mathbf{e}}_{u}, \widehat{\mathbf{e}}_{\phi}$ являются единичными касательными векторами, соответствующими координатам $r, u, \phi$. Плотность тока $\mathbf{J}$ есть

$$
\mathbf{J}=\frac{1}{\mu} \operatorname{curl} \mathbf{B}=\frac{1}{\mu}\left(\frac{I_{r}}{r} \widehat{\mathbf{e}}_{u}-\frac{I_{u}}{r} \widehat{\mathbf{e}}_{r}+\Phi \widehat{\mathbf{e}}_{\phi}\right),
$$

где

$$
\begin{gathered}
I=\frac{r^{2}+\gamma^{2}}{r^{2}} f-\frac{\gamma}{r} \psi_{r} \\
\Phi=\frac{1}{r} \frac{\partial^{2} \psi}{\partial u^{2}}+\frac{\partial}{\partial r}\left(\frac{1}{r} \frac{\partial \psi}{\partial r}\right)-\gamma \frac{\partial}{\partial r}\left(\frac{f}{r^{2}}\right) .
\end{gathered}
$$

Джонсон, Фриман, Калсруд и Оберман показали в работе [38], что уравнения равновесия плазмы (1.1) для решений с винтовой симметрией эквивалентны равенствам $I=I(\psi), p=p(\psi)$ с произвольными функциями $I(\psi)$ и $p(\psi)$ и уравнению

$$
\frac{1}{r^{2}} \frac{\partial^{2} \psi}{\partial u^{2}}+\frac{1}{r} \frac{\partial}{\partial r}\left(\frac{r}{r^{2}+\gamma^{2}} \frac{\partial \psi}{\partial r}\right)+\frac{I I^{\prime}(\psi)}{r^{2}+\gamma^{2}}+\frac{2 \gamma I(\psi)}{\left(r^{2}+\gamma^{2}\right)^{2}}=-\mu p^{\prime}(\psi),
$$


где штрих означает дифференцирование по отношению к $\psi$. Уравнение (7.5) эквивалентно уравнению Джонсона-Фримана-Калсруда-Обермана (ДжФКО) [38], где использовалась другая переменная $u=m \phi-h z$. Мы используем переменную $u=z-\gamma \phi$, потому что только параметр $\gamma=m / h$ является сушественньм. При $\gamma=0$ уравнение Дж$\Phi$ КО (7.5) преврашается в уравнение Грэда-Шафранова (2.3) [4], [5], которое описывает осесимметричные равновесия плазмы.

Формула (7.3) при $I=I(\psi)$ дает

$$
f=\frac{\gamma r \psi_{r}+r^{2} I(\psi)}{r^{2}+\gamma^{2}}, \quad \psi_{r}-\frac{\gamma f}{r}=\frac{r^{2} \psi_{r}-\gamma r I(\psi)}{r^{2}+\gamma^{2}}
$$

Из формул (7.1) и (7.6) следуют выражения для магнитного поля в цилиндрических координатах $r, z, \phi$ :

$$
\mathbf{B}=\frac{\psi_{u}}{r} \widehat{\mathbf{e}}_{r}+B_{1} \widehat{\mathbf{e}}_{z}+B_{2} \widehat{\mathbf{e}}_{\phi}, \quad B_{1}=\frac{\gamma I(\psi)-r \psi_{r}}{r^{2}+\gamma^{2}}, \quad B_{2}=\frac{r I(\psi)+\gamma \psi_{r}}{r^{2}+\gamma^{2}}
$$

и в декартовых координатах $x, y, z$ :

$$
\mathbf{B}=\left(\frac{x \psi_{u}}{r^{2}}-\frac{y\left(\gamma \psi_{r}+r I(\psi)\right)}{r\left(r^{2}+\gamma^{2}\right)}\right) \widehat{\mathbf{e}}_{x}+\left(\frac{y \psi_{u}}{r^{2}}+\frac{x\left(\gamma \psi_{r}+r I(\psi)\right)}{r\left(r^{2}+\gamma^{2}\right)}\right) \widehat{\mathbf{e}}_{y}+B_{1} \widehat{\mathbf{e}}_{z}
$$

Уравнение ДжфКО (7.5) и первая формула (7.6) дают выражение для функции $\Phi / r=-\mu p^{\prime}(\psi)-f I^{\prime}(\psi) / r^{2}$. Отсюда мы получаем, что ток $\mathbf{J}(7.2)$ в цилиндрических координатах $r, z, \phi$ имеет вид

$$
\mathbf{J}=-\frac{I^{\prime}(\psi)}{\mu} \mathbf{B}-p^{\prime}(\psi)\left(r \widehat{\mathbf{e}}_{\phi}+\gamma \widehat{\mathbf{e}}_{z}\right)
$$

Теорема 2. Существует бесконечное семейство глобальных равновесий плазмы, которые зависят от произвольного иелого числа $N \geqslant 0$ и двух вещественных чисел $\beta>0$ и $\gamma$. Эти равновесия являются $z$ - и ф-инвариантнылми в чилиндрических координатах $r, z, \phi$. Для

$$
\beta=\frac{m^{2}}{4 \gamma^{2}(2 N-2 n-m)}
$$

и любых двух иельх чисел $m \geqslant 1$ и $n \geqslant 0$, удовлетворяющих условию $2 N>2 n+m$, эти равновесия содержатся в трехмерном линейном пространстве точных глобальньх равновесий плазмь, которье обладают винтовой симметрией с параметром $\gamma$. 
ДокАЗАТЕЛЬСтво. 1) Предположим, что $I(\psi)=\alpha \psi$ и $p(\psi)=p_{1}-2 \beta^{2} \psi^{2} / \mu$, где $p_{1}>\left(2 \beta^{2} / \mu\right) \max \left(\psi^{2}(x, y, z)\right)$. Тогда давление плазмы $p>0$ всюду в $\mathbb{R}^{3}$. Очевидно, что постоянная $p_{1}$ не входит в уравнения равновесия плазмы. При этих предположениях уравнение Дж $\Phi$ КО (7.5) становится линейньп:

$$
\frac{1}{r^{2}} \frac{\partial^{2} \psi}{\partial u^{2}}+\frac{1}{r} \frac{\partial}{\partial r}\left(\frac{r}{r^{2}+\gamma^{2}} \frac{\partial \psi}{\partial r}\right)=4 \beta^{2} \psi-\frac{\alpha^{2} \psi}{r^{2}+\gamma^{2}}-\frac{2 \alpha \gamma \psi}{\left(r^{2}+\gamma^{2}\right)^{2}} .
$$

Разделяя переменные с помощью подстановки

$$
\psi(r, u)=A(r)(a \cos (\omega u)+b \sin (\omega u)),
$$

мы получаем уравнение

$$
\frac{1}{r} \frac{d}{d r}\left(\frac{r}{r^{2}+\gamma^{2}} \frac{d A(r)}{d r}\right)=\left(4 \beta^{2}+\frac{\omega^{2}}{r^{2}}-\frac{\alpha^{2}}{r^{2}+\gamma^{2}}-\frac{2 \alpha \gamma}{\left(r^{2}+\gamma^{2}\right)^{2}}\right) A(r) .
$$

Это уравнение не принадлежит никакому известному классу интегрируемых уравнений, см. [57]. Для нахождения точных решений мы применим подстановку $A(r)=$ $r^{\lambda} e^{-\beta r^{2}} B(x), x=2 \beta r^{2}, \lambda \geqslant 0$, которая преобразует уравнение (7.12) в уравнение

$$
\begin{aligned}
& \left(x^{2}+c_{1} x\right) B^{\prime \prime}+\left(-x^{2}+\left(\lambda-c_{1}\right) x+(\lambda+1) c_{1}\right) B^{\prime} \\
& \quad+\left(\frac{\alpha^{2}-\eta}{8 \beta} x+\frac{\alpha^{2} \gamma^{2}-\eta \gamma^{2}}{4}+\frac{\alpha \gamma-c_{1}-\lambda}{2}+\frac{x+c_{1}}{4 x}\left(\lambda^{2}-\gamma^{2} \omega^{2}\right)\right) B=0,
\end{aligned}
$$

где $B^{\prime}=d B(x) / d x, c_{1}=2 \beta \gamma^{2}$ и $\eta=4 \beta^{2} \gamma^{2}+4 \beta \lambda+\omega^{2}$. Мы интересуемся только полиномиальными решениями $B(x)$ с ненулевым свободным членом. Исследуя члены высшего и низшего порядков в (7.13), мы получаем необходимые условия

$$
\frac{\alpha^{2}-\eta}{8 \beta}=n, \quad \lambda=|\gamma \omega|,
$$

где целое число $n \geqslant 0$ является степеню многочлена $B(x)$. Форма решения (7.11)

$$
\psi(r, u)=A(r)(a \cos (\omega z-\gamma \omega \phi)+b \sin (\omega z-\gamma \omega \phi))
$$

имеет следствием то, что $\gamma \omega$ обязано быть целым числом: $|\gamma \omega|=m \geqslant 0$. Отсюда мы получаем $\lambda=m, \omega= \pm m / \gamma$ и $\eta=(2 \beta \gamma+m / \gamma)^{2}$. Первое необходимое условие (7.14) становится уравнением

$$
\alpha^{2} \gamma^{2}=\left(m+c_{1}\right)^{2}+4 n c_{1}
$$

для двух неизвестных целых чисел $m$ и $n$.

Представим уравнение (7.13) в виде

(7.16) $\left(x^{2}+c_{1} x\right) B^{\prime \prime}+\left(-x^{2}+\left(m-c_{1}\right) x+(m+1) c_{1}\right) B^{\prime}+n\left(x+c_{1}-k_{m n} c_{1}\right) B=0$,

где $k_{m n}=\left(m+c_{1}-\alpha \gamma\right) /\left(2 n c_{1}\right)$. Отметим, что уравнение (7.16) отличается от всех классических дифференциальных уравнений, которые определяют полиномы Чебышёва, Эрмита, Лагерра, Лежандра и Якоби [55]-[57]. 
ЛЕмма 1. Дифференциальное уравнение (7.16) при выполнении условия (7.15) имеет полиномиальное решение

$$
B_{m n}(x)=\frac{d^{m}}{d x^{m}} L_{m+n}(x)-k_{m n} x \frac{d^{m+1}}{d x^{m+1}} L_{m+n}(x),
$$

әде $L_{m}(x)$ являются полиномами Лагерра.

ДокАЗАТЕльСтво. Для нахождения полиномиальных решений $B_{m n}(x)$ мы сделаем подстановку

$$
B(x)=P(x)-k_{m n} x P^{\prime}(x) .
$$

Прямая проверка доказывает тождество

$$
\begin{aligned}
& \left(x^{2}+c_{1} x\right) B^{\prime \prime}+\left(-x^{2}+\left(m-c_{1}\right) x+(m+1) c_{1}\right) B^{\prime}+n\left(x+c_{1}-k_{m n} c_{1}\right) B \\
& \quad=\left(x+c_{1}-k_{m n} c_{1}\right) Q-k_{m n} x\left(x+c_{1}\right) Q^{\prime}+\left(n c_{1} k_{m n}^{2}-\left(m+c_{1}\right) k_{m n}-1\right) x P^{\prime}
\end{aligned}
$$

где $Q=x P^{\prime \prime}+(1+m-x) P^{\prime}+n P$. Необходимое условие (7.15) эквивалентно уравнению

$$
n c_{1} k_{m n}^{2}-\left(m+c_{1}\right) k_{m n}-1=0, \quad k_{m n}=\frac{m+c_{1}-\alpha \gamma}{2 n c_{1}} .
$$

Уравнение $Q=0$, или

$$
x P^{\prime \prime}+(1+m-x) P^{\prime}+n P=0,
$$

имеет полиномиальные решения

$$
P_{m n}(x)=\frac{d^{m} L_{m+n}(x)}{d x^{m}}
$$

степени $n$, где $L_{p}(x)$ - полиномы Лагерра

$$
L_{p}(x)=\frac{1}{p !} e^{x} \frac{d^{p}}{d x^{p}}\left(e^{-x} x^{p}\right)=\sum_{k=0}^{p} \frac{(-1)^{k} p !}{(k !)^{2}(p-k) !} x^{k} .
$$

Отсюда, используя формулу (7.18), мы получаем, что если два целых числа $m$ и $n$ удовлетворяют уравнению (7.15), то уравнение (7.16) следует из уравнения $Q=0$ и имеет полиномиальное решение (7.17).

Окончательно мы получаем, что уравнение (7.10) имеет точное решение

$$
\psi_{m n}=r^{m} e^{-\beta r^{2}} B_{m n}\left(2 \beta r^{2}\right)\left(a_{m n} \cos (m u / \gamma)+b_{m n} \sin (m u / \gamma)\right),
$$

где $a_{m n}$ и $b_{m n}$ являются произвольньми постоянными. При $m \geqslant 2$ магнитное поле (7.7) $\mathbf{B}(0, z)=0$ на оси $r=0$. Только при $m=1$ кинк-мода (7.20) имеет ненулевое магнитное поле при $r=0$.

В силу линейности уравнения (7.10) мы получаем, что если алгебраическое уравнение (7.15) имеет несколько целых решений $m, n$, то любая линейная комбинация соответствуюших функций (7.20) является точньм решением уравнения (7.10). 
2) При $m=n=0$ необходимое условие (7.15) принимает вид $\alpha=2 \beta \gamma$ и уравнение (7.13) имеет решение $B=$ const. Поэтому уравнение ДжфКО (7.5) имеет точное решение гауссовского вида

$$
\psi_{0}(r)=\exp \left(-\beta r^{2}\right)
$$

Соответствующее магнитное поле (7.7), ток (7.8) и давление $p$ имеют вид

$$
\mathbf{B}=2 \beta e^{-\beta r^{2}} \widehat{\mathbf{e}}_{z}, \quad \mathbf{J}=\frac{4 \beta^{2}}{\mu} r e^{-\beta r^{2}} \widehat{\mathbf{e}}_{\phi}, \quad p=p_{1}-\frac{2 \beta^{2}}{\mu} e^{-2 \beta r^{2}} .
$$

Это некоторое глобальное равновесие плазмы, обладающее цилиндрической симметрией.

Для $m=0$ и $n=N \neq 0$ необходимое условие (7.15) сводится к уравнению $\left(c_{1}=2 \beta \gamma^{2}\right)$

$$
\alpha^{2} \gamma^{2}=c_{1}^{2}+4 N c_{1}
$$

и точное решение (7.20) принимает вид

$$
\psi_{N}(r)=a_{N} e^{-\beta r^{2}} B_{0 N}\left(2 \beta r^{2}\right),
$$

где многочлены $B_{0 N}(x)$ определяются формулами

$$
B_{0 N}(x)=L_{N}(x)-k_{N} x L_{N}^{\prime}(x) .
$$

Из уравнения (7.23) следует

$$
\alpha \gamma=c_{1} \sqrt{1+2 N /\left(\beta \gamma^{2}\right)}, \quad k_{N}=\frac{c_{1}-\alpha \gamma}{2 N c_{1}}=\frac{1-\sqrt{1+2 N /\left(\beta \gamma^{2}\right)}}{2 N}<0 .
$$

Поэтому многочлены $B_{0 N}(x)$ зависят от параметра $\beta \gamma^{2}$.

Для функции магнитного потока (7.24) магнитное поле (7.7)

$$
\mathbf{B}=\frac{\alpha \gamma \psi_{N}-r \psi_{N}^{\prime}}{r^{2}+\gamma^{2}} \widehat{\mathbf{e}}_{z}+\frac{\alpha r \psi_{N}+\gamma \psi_{N}^{\prime}}{r^{2}+\gamma^{2}} \widehat{\mathbf{e}}_{\phi}
$$

не имеет сингулярностей и убывает при $r \rightarrow \infty$ так же быстро, как $\exp \left(-\beta r^{2}\right) r^{2 N}$. Поэтому полная магнитная энергия в любом слое $r_{1} \leqslant r \leqslant r_{2}$ является конечной. Магнитные поверхности $\psi_{N}(r)=$ const являются цилиндрами $r=$ const.

Таким образом, мы продемонстрировали, что уравнение Дж $\Phi \mathrm{KO}(7.5)$ имеет решения, описываюшие глобальные равновесия плазмы $(7.21),(7.22)$ и $(7.24),(7.27)$, где два параметра $\beta>0$ и $\gamma \neq 0$ являются произвольными. Эти равновесия обладают цилиндрической симметрией, поскольку они являются $z$ - и $\phi$-инвариантными.

3) При $m \geqslant 1$ функции магнитного потока $\psi_{m n}(r, u)(7.20)$ определяют магнитные поля $\mathbf{B}(7.7)$ и токи $\mathbf{J}(7.8)$, которые являются гладкими в декартовых координатах и убывают при $r \rightarrow \infty$ так же быстро, как $\exp \left(-\beta r^{2}\right) r^{2 m}$. Поэтому вьшеуказанные условия а) и b) выполнены. Однако условие с) не удовлетворено, поскольку магнитная 
поверхность $\psi_{m n}(r, u)=0$ есть геликоид $z=\gamma \phi+c$, который является неограниченным по переменной $r$.

Для нахождения глобальных равновесий плазмы мы рассмотрим линейную комбинацию точных решений $\psi_{N}(r)(7.24)$ и $\psi_{m n}(r, u)(7.20)$, которая удовлетворяет уравнению (7.10), если два необходимых условия (7.15) и (7.23) справедливы одновременно. Из этих двух условий следует неравенство $2 N>2 n+m$ и формулы

$$
\begin{aligned}
c_{1} & =2 \beta \gamma^{2}=\frac{m^{2}}{2(2 N-2 n-m)}, \quad \alpha \gamma=\frac{m \sqrt{(4 N-m)^{2}-16 n N}}{2(2 N-2 n-m)} \\
k_{m n} & =\frac{m+c_{1}-\alpha \gamma}{2 n c_{1}}=\frac{1}{2 m n}\left(4(N-n)-m-\sqrt{(4 N-m)^{2}-16 n N}\right)
\end{aligned}
$$

Первая формула (7.28) эквивалентна условию (7.9). Таким образом, мы получаем, что уравнение ДжфКО (7.5) для любых значений параметров $\gamma$ и $\alpha(\gamma), \beta(\gamma)$, определенных формулами (7.28), имеет точные решения

$$
\psi_{N m n}=e^{-\beta r^{2}}\left(a_{N} B_{0 N}(x)+r^{m} B_{m n}(x)\left(a_{m n} \cos (m u / \gamma)+b_{m n} \sin (m u / \gamma)\right)\right)
$$

где $N, m, n$-произвольные целые числа, удовлетворяюшие неравенству $2 N>2 n+m$, и $x=2 \beta r^{2}$. Функции $I(\psi)$ и $p(\psi)$ имеют вид $I(\psi)=\alpha \psi, p(\psi)=p_{1}-2 \beta^{2} \psi^{2} / \mu$, где параметры $\alpha(\gamma), \beta(\gamma)$ заданы формулами (7.28). Из неравенства $2 N>2 n+m$ следует, что функция $\psi_{N m n}(r, u)(7.30)$ имеет главный член $(-2 \beta)^{N} a_{N} \exp \left(-\beta r^{2}\right) r^{2 N}$ при $r \rightarrow \infty$. Поэтому равновесие (7.30) при $m \geqslant 1$ удовлетворяет вышеуказанньм условиям а) и $\mathrm{b})$, и все магнитные поверхности $\psi(r, u)=$ const асимптотически при $r \gg 1$ являются цилиндрами $r=$ const. Поэтому все магнитные силовые линии и линии тока являются ограниченными по переменной $r$. Таким образом, точные решения (7.30) определяют глобальные равновесия плазмы. Эти равновесия являются периодическими по переменной $z$ с периодом $2 \pi \gamma / m$ и по переменной $\phi$ с периодом $2 \pi / m$. При $m \geqslant 1$ точные решения (7.30) описьвают сильно сконцентрированные вдоль оси $z$ кинк-моды в "прямом токамаке".

Из уравнения (7.15) при $\alpha>0$ следует $m+c_{1}<\alpha \gamma$, поэтому $k_{m n}<0$. Отсюда, используя классические свойства полиномов Лагерра (7.19), мы получаем, что новые многочлены $B_{m n}(x)(7.17)$ имеют знакопеременные коэффициенты и каждый многочлен $B_{m n}(x)$ имеет $n$ различных положительных корней при любом целом $m \geqslant 0$.

Используя формулы (7.19), мы находим первые три многочлена (7.25):

$$
\begin{gathered}
B_{01}(x)=1-\left(1-k_{1}\right) x, \quad B_{02}(x)=1-2\left(1-k_{2}\right) x+\frac{1}{2}\left(1-2 k_{2}\right) x^{2}, \\
B_{03}(x)=1-3\left(1-k_{3}\right) x+\frac{3}{2}\left(1-2 k_{3}\right) x^{2}-\frac{1}{6}\left(1-3 k_{3}\right) x^{3} .
\end{gathered}
$$

Первые три многочлена $B_{1 n}(x)(7.17)$ имеют вид

$$
\begin{aligned}
& B_{10}(x)=-1, \quad B_{11}(x)=-2+\left(1-k_{11}\right) x \\
& B_{12}(x)=-3+3\left(1-k_{12}\right) x-\frac{1}{2}\left(1-2 k_{12}\right) x^{2}
\end{aligned}
$$




\section{§ 8. Модель астрофизических струй}

ПримеР 4. При $N=0$ сушествует только одно равновесие плазмы $\psi_{0}(x)=$ $c \exp \left(-\beta r^{2}\right)$. При $N=1$ неравенство $2 N>2 n+m$ имеет одно целочисленное решение $m=1, n=0$. Формулы (7.28) дают $c_{1}=1 / 2, \alpha \gamma=3 / 2, \beta=1 /\left(4 \gamma^{2}\right)$; отсюда $k_{1}=-1$ и многочлен $B_{01}(x)$ (7.31) есть $1-2 x$. Таким образом, функция магнитного потока (7.30) имеет эквивалентный вид $\left(x=2 \beta r^{2}\right)$

$$
\psi(r, z, \phi)=e^{-\beta r^{2}}\left(1-4 \beta r^{2}+a_{1} r \cos (z / \gamma-\phi)\right) .
$$

Рис. 7 показьвает сечение $z=0$ магнитных поверхностей $\psi(r, z, \phi)=$ const при $a_{1}=-1, \beta=0.1, \gamma=\sqrt{5 / 2}$. Функция $\psi(r, 0, \phi)(8.1)$ достигает своего максимума при $r=0.8968, \phi=\pi$ и минимума при $r=3.0168, \phi=0$.

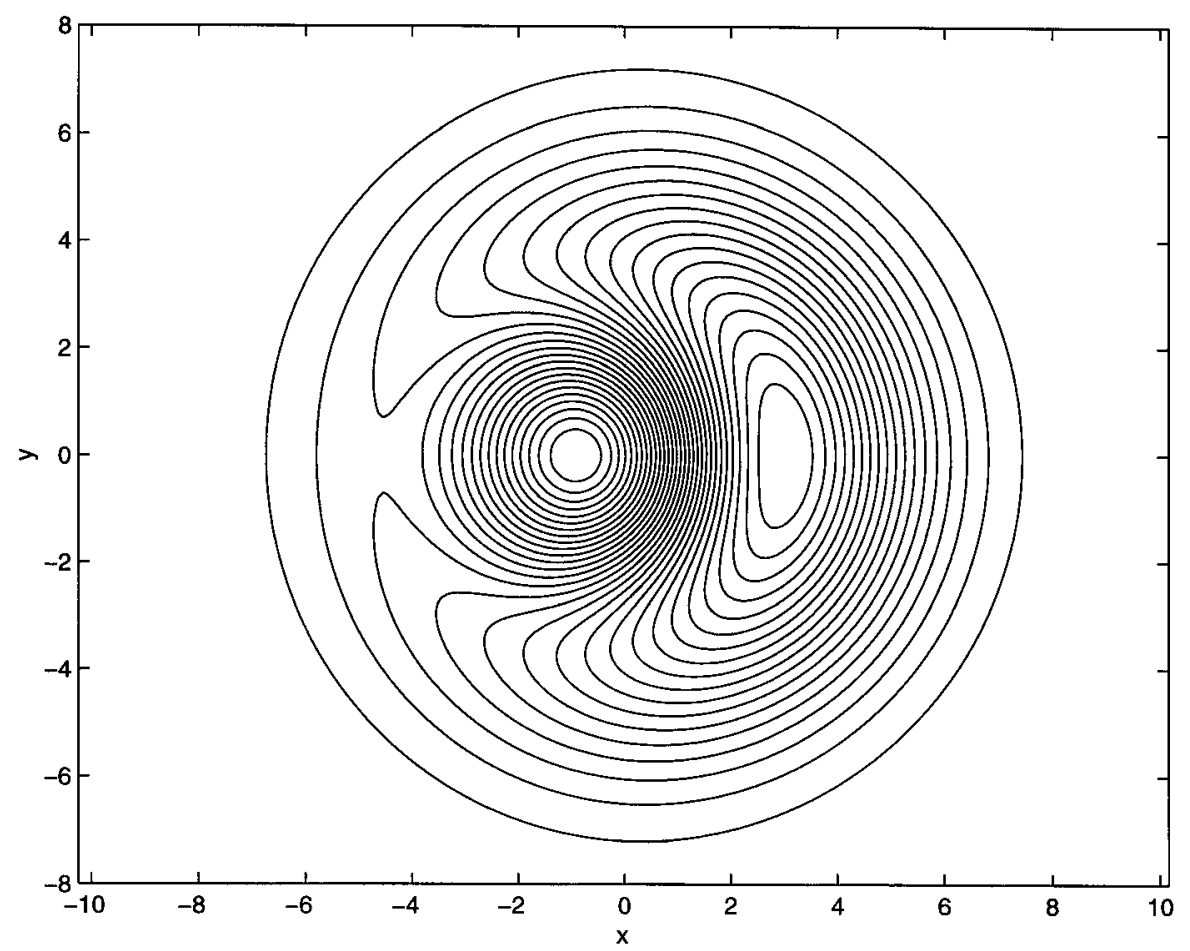

Рис. 7. Магнитные поверхности при $z=0$ для винтовой модели астрофизических струй

Двумерные магнитные поверхности получаются из кривых на рис. 7 посредством одновременного врашения по $\phi$ с угловой скоростью 1 и сдвига по $z$ с угловой скоростью $\gamma$. Рис. 7 показывает, что существуют три области, заполненные цилиндрическими магнитньми поверхностями: одна внешшяя область и две внутренние области с двумя магнитньми осями, соответствующими критическим точкам максимума и минимума функции $\psi(r, u)$. Эти магнитные оси образуют двойную спираль, представленную на рис. 8. Магнитные оси являются точными магнитными силовыми линиями. 


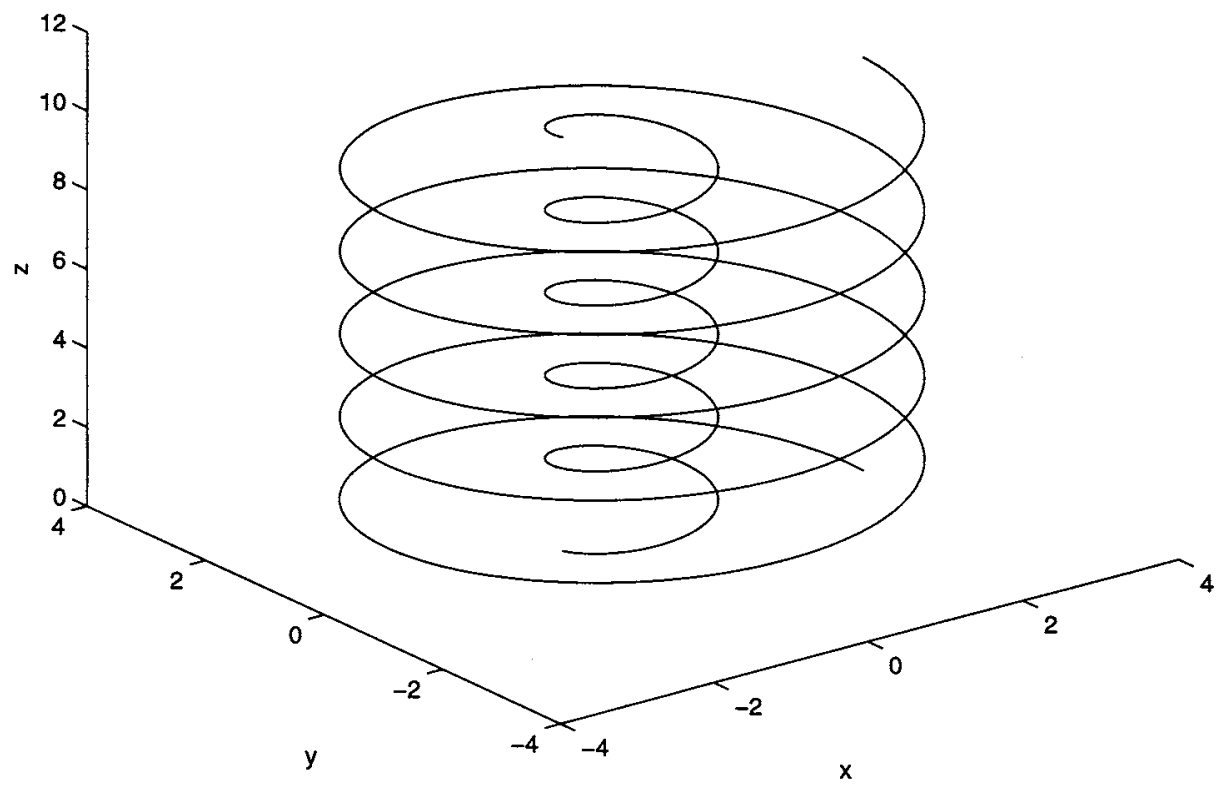

Рис. 8. Двойная спираль магнитных осей

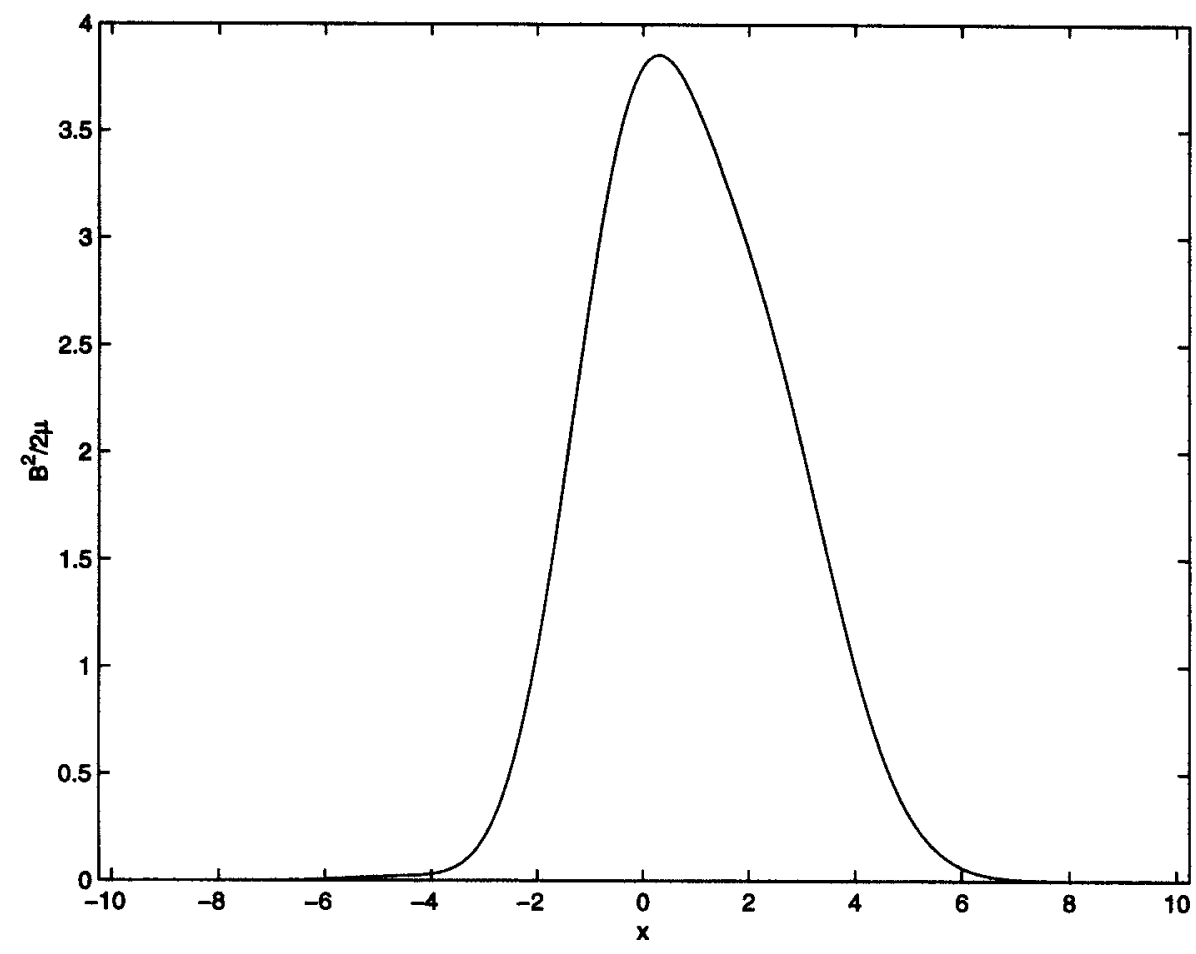

Рис. 9. Плотность магнитной энергии $B^{2}(x, 0,0) /(2 \mu)$ 


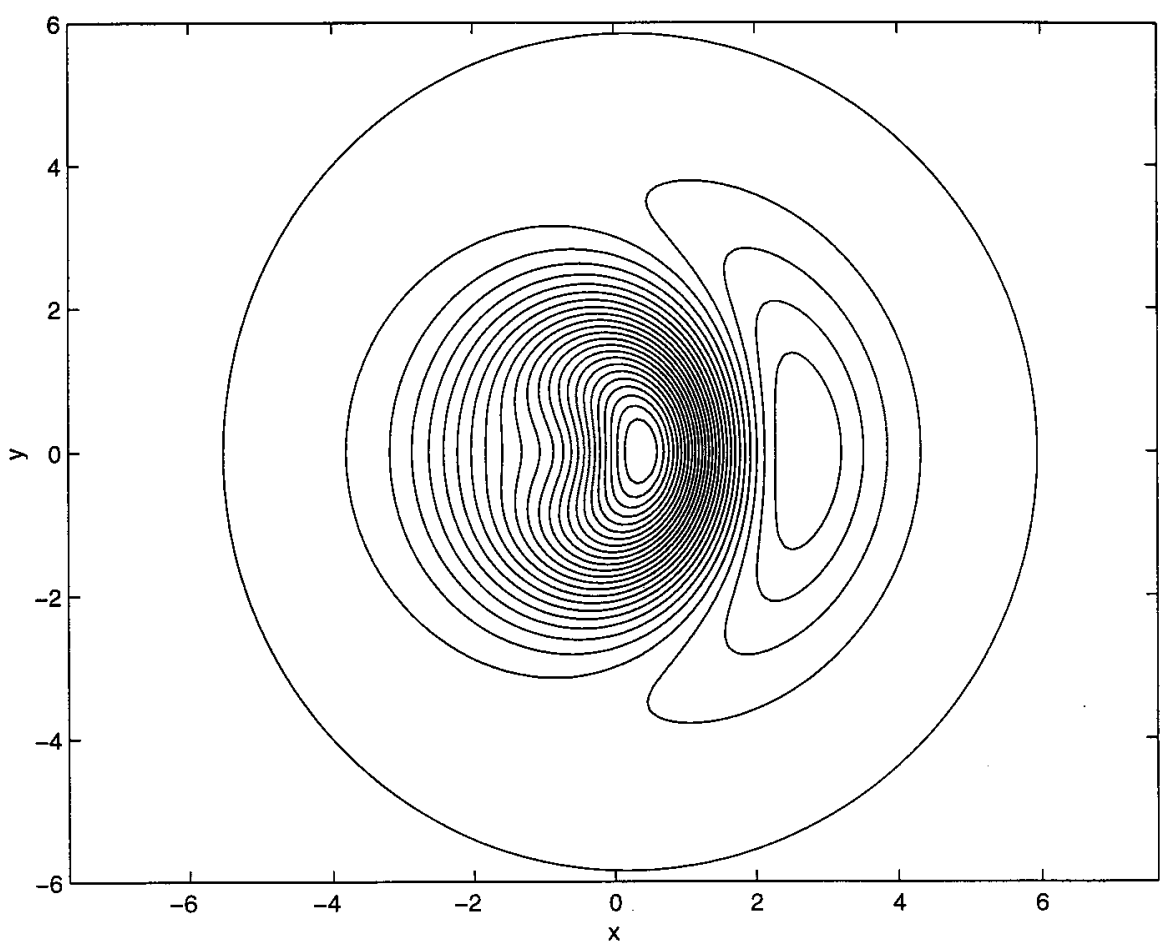

Рис. 10. Поверхности постоянной магнитной энергии $B^{2}(x, y, z)=$ const при $z=0$

Рис. 9 представляет плотность магнитной энергии $B^{2}(x, y, z) /(2 \mu)$ для равновесия плазмы (8.1) при $y=0, z=0$ и $a_{1}=-1, \beta=0.1, \gamma=\sqrt{5 / 2}, \mu=0.1$. Рис. 10 показьвает линии уровня $B^{2}(x, y, 0)=$ const, $z=0$. Магнитная энергия сконцентрирована около оси $z$ и очень быстро стремится к нулю при $r \rightarrow \infty$. Это свойство означает, что вьшшеуказанное точное решение моделирует сильно сконцентрированную астрофизическую струю в сопутствующей системе отсчета. Наблюдаемый вид струи определяется ее синхротронным излучением, которое пропорционально $B^{2}$ [35]. Поэтому детектируемая форма струи приближенно совпадает с некоторой поверхностью $B^{2}(x, y, z)=$ const. Поверхности $B^{2}=$ const получаются из кривых на рис. $10 \mathrm{c}$ помощью винтовых преобразований. Из рис. 7 и рис. 10 с очевидностью следует, что магнитные поверхности $\psi=$ const и поверхности $B^{2}=$ const являются различными.

Рис. 11 показывает давление плазмы $p(x, y, z)=p_{1}-2 \beta^{2} \psi^{2} / \mu$ при $y=z=0$, $p_{1}=1,2$ для равновесия плазмы (8.1). Графики на рис. 9 и 11, очевидно, являются асимметричными по отношению к $x=0$.

ПримеР 5 . При $N=2$ неравенство $2 N>2 n+m$ имеет четыре целых решения $m=1, n=0 ; m=1, n=1 ; m=2, n=0$ и $m=3, n=0$. Для $m=1, n=0$ мы находим из формул (7.28): $c_{1}=1 / 6, \alpha \gamma=7 / 6, \beta=1 /\left(12 \gamma^{2}\right)$. Поэтому $k_{2}=-3 / 2$ и многочлен $B_{02}(x)(7.31)$ есть $1-5 x+2 x^{2}$. Точное решение (7.30) имеет эквивалентньй вид

$$
\psi(r, z, \phi)=e^{-\beta r^{2}}\left(1-10 \beta r^{2}+8 \beta^{2} r^{4}+a_{1} r \cos (z / \gamma-\phi)\right) .
$$




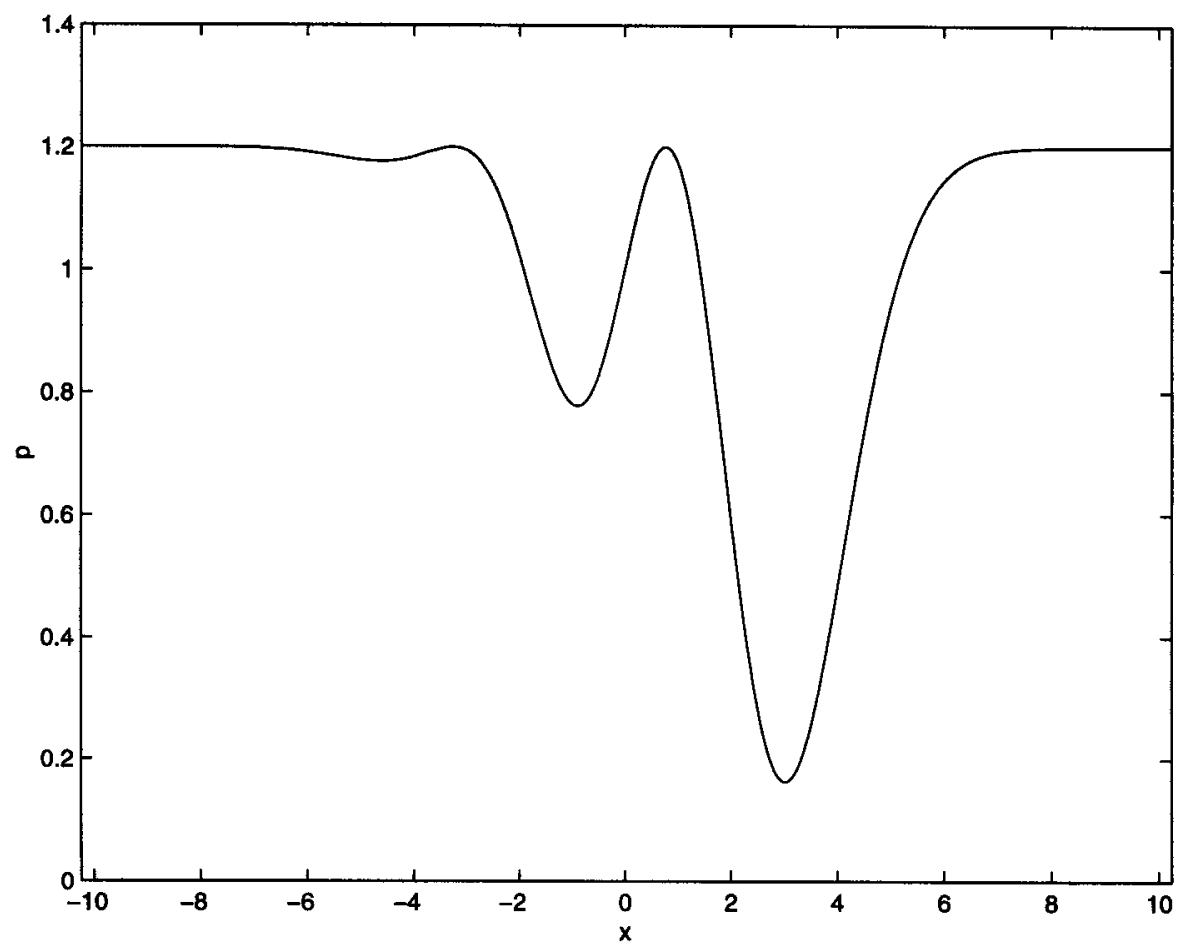

Рис. 11. Давление плазмы $p(x, 0,0)$ для винтовой модели астрофизических струй

Сечение $z=0$ магнитных поверхностей $\psi(r, z, \phi)=$ const показано на рис. 12 для $a_{1}=-1.5, \beta=0.1, \gamma=\sqrt{5 / 6}$. Функция магнитного потока $\psi(r, 0, \phi)(8.2)$ достигает своего локального максимума в двух точках $r=4.8625, \phi=\pi$ и $r=0.6940, \phi=\pi$ и своего минимума при $r=2.2361, \phi=0$. Поэтому имеются четыре инвариантные области в $\mathbb{R}^{3}$ заполненные цилиндрическими магнитными поверхностями: одна внешняя область и три внутренние области. Соответствующие три магнитные оси образуют тройную спираль, которая изображена на рис. 13. Кривые на рис. 7 и рис. 12 симметричны по отношению к отражению $y \rightarrow-y$.

\section{§. Квазипериодичность магнитных силовых линий}

В координатах $r, z, \phi$ магнитные поверхности $\psi(r, u)=h=$ const являются врашающимися винтовыми цилиндрами $C_{h}$, см. рис. 7. Сечения $S_{k}: z=2 \pi \gamma k$ цилиндра $C_{h}$ являются одинаковыми для всех целых чисел $k$ в силу периодичности $z \rightarrow z+2 \pi \gamma$ функции магнитного потока (7.30). Поэтому последовательные пересечения магнитных силовых линий на $C_{h}$ с кривыми $S_{0}$ и $S_{1}$ определяют преобразование врашения $T_{h}: S_{0} \rightarrow S_{0}=S_{1}$. Для подходяшего углового параметра $s=s \bmod 1$ на замкнутой кривой $S_{0}$ отображение $T_{h}$ есть $T_{h}(s)=s+\theta_{0}(h)$. Для обших $h$ число врашения $\theta_{0}(h)$ является иррациональным. Поэтому общие магнитные силовые линии являются квазипериодическими в переменных $r, z, \phi$.

Мы докажем это общее свойство для тонких эллиптических цилиндров $C_{h}$, враша- 


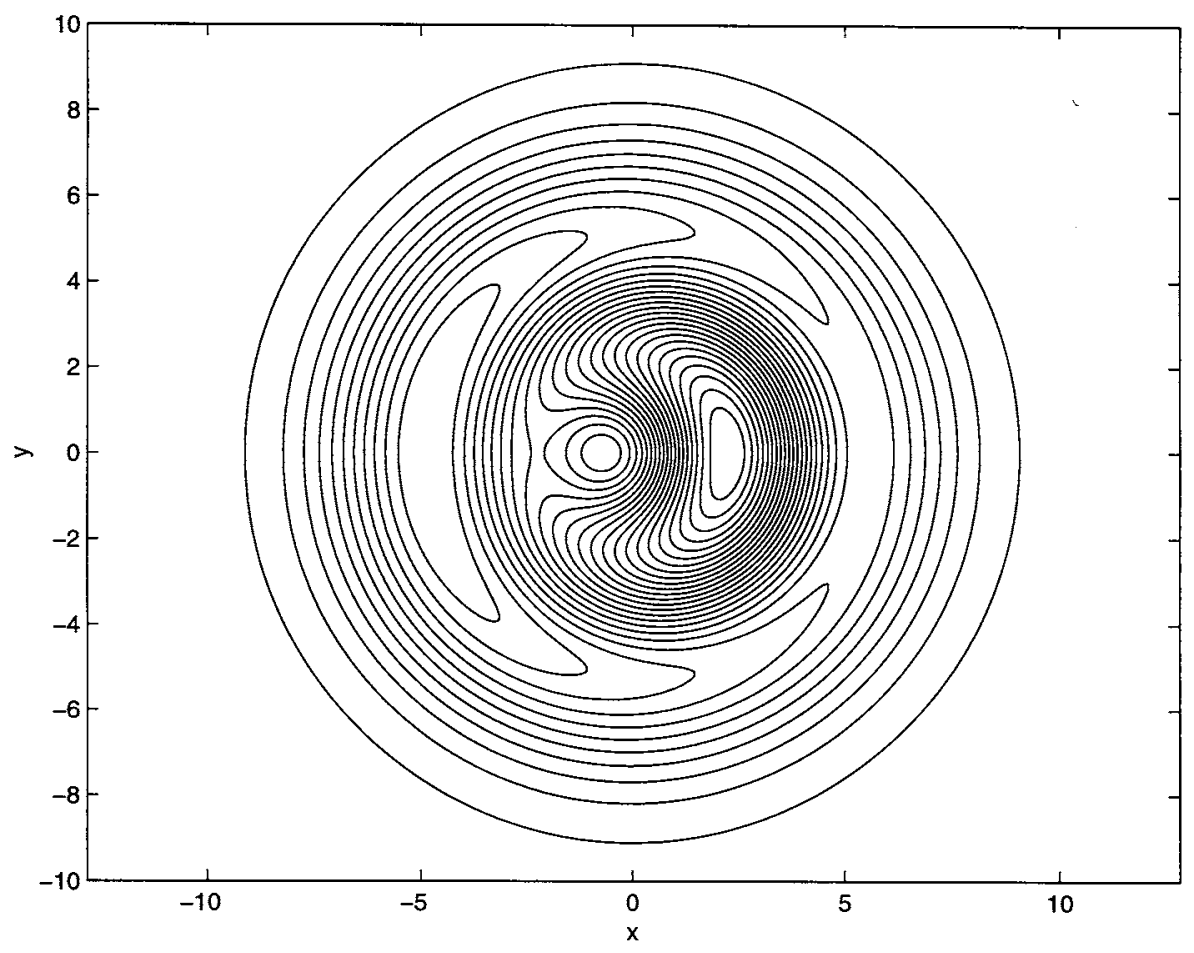

Рис. 12. Магнитные поверхности для равновесия плазмы (8.2) при $z=0$

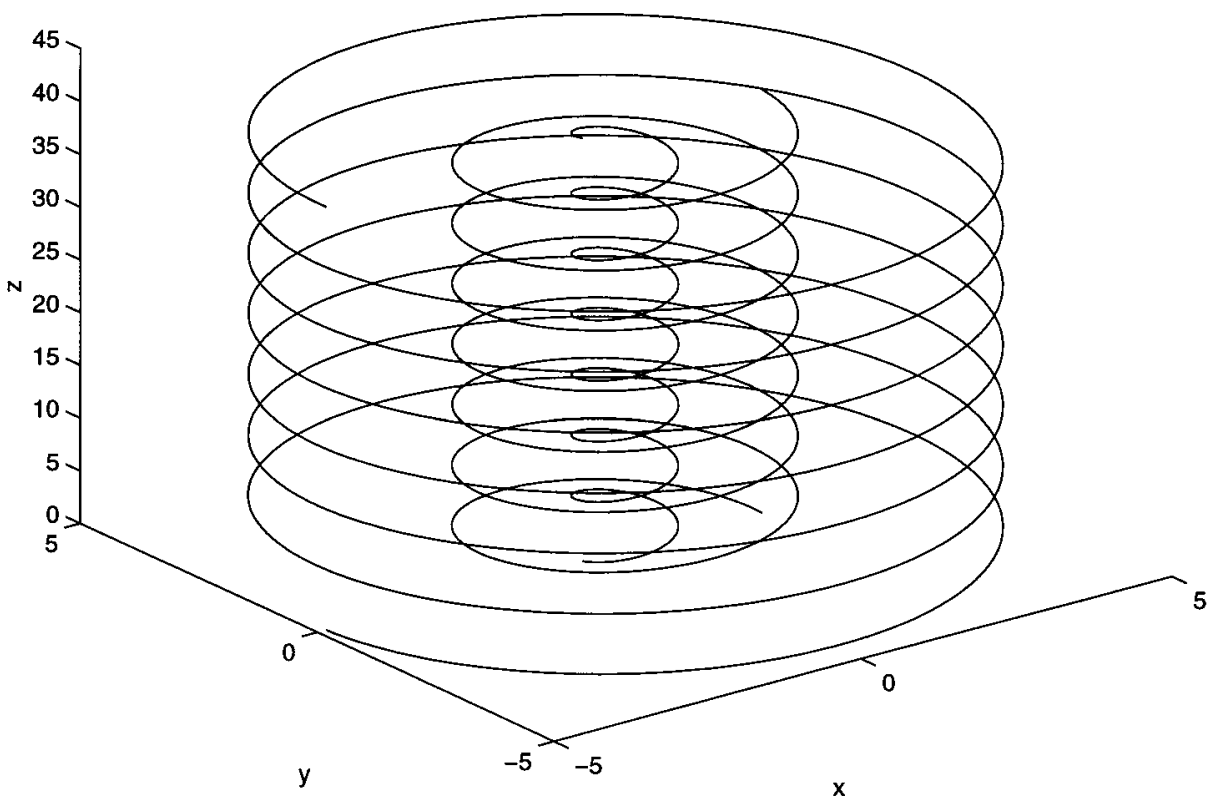

Рис. 13. Тройная спираль магнитных осей 
ющихся вокруг их магнитных осей. Пусть некоторое точное решение (7.30) достигает своего локального максимума (или минимума) в некоторой точке $\left(r_{0}, u_{0}\right): \psi\left(r_{0}, u_{0}\right)=$ $\psi_{0}$. Критическая точка $\left(r_{0}, u_{0}\right)$ определяет магнитную ось $M_{0}: r=r_{0}, z-\gamma \phi=u_{0}$. Во втором приближении имеем

$$
\psi\left(r_{0}+\delta r, u_{0}+\delta u\right) \approx \psi_{0}+a(\delta r)^{2}+b(\delta u)^{2}+2 c \delta r \delta u
$$

где $2 a=\psi_{r r}\left(r_{0}, u_{0}\right), 2 b=\psi_{u u}\left(r_{0}, u_{0}\right), 2 c=\psi_{r u}\left(r_{0}, u_{0}\right)$. Для точек $\left(r_{0}, u_{0}\right)$ максимума или минимума мы имеем $\psi_{r r} \psi_{u u}-\psi_{r u}^{2} \geqslant 0$. В малой окрестности спирали $M_{0}$ магнитные поверхности $\psi(r, u)=$ const являются тонкими эллиптическими цилиндрами $C_{h}$, вращающимися вокруг $M_{0}$.

Вычислим число врашения $\theta_{0}$ для кривых $S_{0}$ при $h \ll 1$. Кривая $S_{0}$ аппроксимируется эллипсом

$$
a(\delta r)^{2}+b(\delta u)^{2}+2 c \delta r \delta u=h^{2}
$$

с параметризацией

$$
\delta r=\kappa h \sin (2 \pi s), \quad \delta u=-c \kappa h \sin (2 \pi s) / b+h \cos (2 \pi s) / \sqrt{b},
$$

где $\kappa=\sqrt{b} / \sqrt{a b-c^{2}}$ и $\delta u=-\gamma \delta \phi$. Магнитное поле (7.7) определяет уравнения для магнитных силовых линий

$$
\dot{r}=\frac{\psi_{u}}{r}, \quad \dot{z}=\frac{\alpha \gamma \psi-r \psi_{r}}{r^{2}+\gamma^{2}}, \quad \dot{\phi}=\frac{\alpha r \psi+\gamma \psi_{r}}{r\left(r^{2}+\gamma^{2}\right)} .
$$

На магнитной оси $M_{0}$ мы имеем $z(t)=a \gamma \psi_{0} t /\left(r^{2}+\gamma^{2}\right)+z_{0}$. Поэтому сдвиг по $z$ на период $2 \pi \gamma$ происходит при $t=t_{0}=2 \pi\left(r_{0}^{2}+\gamma^{2}\right) /\left(\alpha \psi_{0}\right)$. Из уравнений (9.1) следуют уравнения для вариаций

$$
r_{0}(\delta r)^{\cdot}=2 b \delta u+2 c \delta r, \quad r_{0}(\delta u)^{\cdot}=-2 a \delta r-2 c \delta u
$$

Их решения имеют вид

$$
\delta r(t)=\kappa h \sin (\omega t+2 \pi s), \quad \delta u(t)=-c \kappa h \sin (\omega t+2 \pi s) / b+h \cos (\omega t+2 \pi s) / \sqrt{b},
$$

где $\omega=2 \sqrt{a b-c^{2}} / r_{0}$. При $t=t_{0}$ мы получаем преобразование врашения $T(s)=s+$ $t_{0} \sqrt{a b-c^{2}} /\left(\pi r_{0}\right)$. Поэтому число врашения есть

$$
\theta_{0}=\frac{\left(r_{0}^{2}+\gamma^{2}\right) \sqrt{\psi_{r r} \psi_{u u}-\psi_{r u}^{2}}}{\alpha r_{0} \psi_{0}} .
$$

В общем положении число врашения $\theta_{0}(9.2)$ иррационально и зависит от малого параметра $h$. Поэтому общие магнитные силовые линии являются квазипериодическими в переменных $r, z, \phi$. Из этого свойства следует, что характер врашения магнитных силовых линий друг вокруг друга изменяется в направлении оси $z$ для выведенных глобальных равновесий плазмы (7.30) и не повторяется. 


\section{$\S$ 10. Винтовые равновесия плазмы, зависящие от $2 K+1$ параметров}

I. Для вывода равновесий плазмы, зависяших от более чем трех параметров, достаточно найти более чем два целочисленных решения алгебраических уравнений (7.15) и (7.23) с данными постоянными $\alpha \gamma$ и $c_{1}$. Из первой формулы (7.28) следует, что параметр $c_{1}=2 \beta \gamma^{2}$ должен быть рациональным, $c_{1}=p / q$. Исключая $\alpha \gamma=\sqrt{c_{1}^{2}+4 N c_{1}}$ из уравнений (7.15) и (7.23), мы получаем диофантово уравнение [39]

$$
4 p N=q m^{2}+2 p m+4 p n .
$$

В обшем случае уравнение (10.1) для заданных $N, p, q$ имеет несколько целочисленных решений $m, n$. Беря линейную комбинацию соответствуюших функций магнитного потока $\psi_{N}(r)(7.24)$ и $\psi_{m n}(r, u)(7.20)$, мы получаем новое точное решение линейного уравнения (7.10). Мы представим эту конструкцию в явном виде для $c_{1}=1 /(2 l)$, где $l$ есть произвольное нечетное число.

ТЕОРемА 3. Цилиндрически-симметричные равновесия плазмы (7.24), (7.27) при $\beta=1 /\left(4 l \gamma^{2}\right)$ содерәсатся в $(2 K+1)$-мерных линейных пространствах глобальных равновесий плазмы, которые обладают винтовой симметрией с параметром $\gamma$. Здесь $K=[(\sqrt{8 l N+1}-1) /(2 l)]$.

ДокАЗАТЕЛЬСтво. При $p=1, q=2 l$ уравнение (10.1) принимает вид

$$
2 N=l m^{2}+m+2 n \text {. }
$$

Очевидно, что это диофантово уравнение имеет $K+1$ пару целочисленных решений

$$
m=0,1,2, \ldots, K, \quad n=N-\frac{m(l m+1)}{2}, \quad K=\left[\frac{\sqrt{8 l N+1}-1}{2 l}\right] .
$$

Коэффициенты $k_{N}(7.26)$ и $k_{m n}(7.29)$ имеют вид

$$
k_{N}=\frac{1-\sqrt{8 l N+1}}{2 N}, \quad k_{m n}=\frac{2 l m+1-\sqrt{8 l N+1}}{2 n} .
$$

Беря линейную комбинацию точных решений $\psi_{N}(r)(7.24)$ и $\psi_{m n}(r, u)(7.20)$ при $1 \leqslant m \leqslant K$, мы получаем, что функция магнитного потока

(10.4) $\psi=e^{-\beta r^{2}}\left(a_{N} B_{0 N}(x)+\sum_{m=1}^{K} r^{m} B_{m n}(x)\left(a_{m n} \cos (m u / \gamma)+b_{m n} \sin (m u / \gamma)\right)\right)$

удовлетворяет уравнению ДжфКО (7.5) и линейно зависит от $2 K+1$ произвольных параметров $a_{N}, a_{m n}, b_{m n}$. Соответствуюшие магнитное поле $\mathbf{B}(7.7)$, ток $\mathbf{J}(7.8)$ и давление $p=p_{1}-2 \beta^{2} \psi^{2} / \mu$ являются гладкими и ограниченными во всем евклидовом пространстве $\mathbb{R}^{3}$. Функция $\psi(r, u)(10.4)$ имеет главньй член $(-2 \beta)^{N} a_{N} \exp \left(-\beta r^{2}\right) r^{2 N}$ при $r \rightarrow \infty$. Поэтому асимптотически при $r \gg 1$, все магнитные поверхности $\psi(r, u)=$ const являются цилиндрами $r=$ const, и магнитное поле $\mathbf{B}$ и ток $\mathbf{J}$ убывают при $r \rightarrow \infty$ так же быстро, как $c_{N} \exp \left(-\beta r^{2}\right) r^{2 N}$, и $p \rightarrow p_{1}$. Поэтому функции магнитного потока $\psi(r, u)(10.4)$ определяют глобальные равновесия плазмы, удовлетворяюшие вьшеуказанным физическим условиям a), b) и с). 


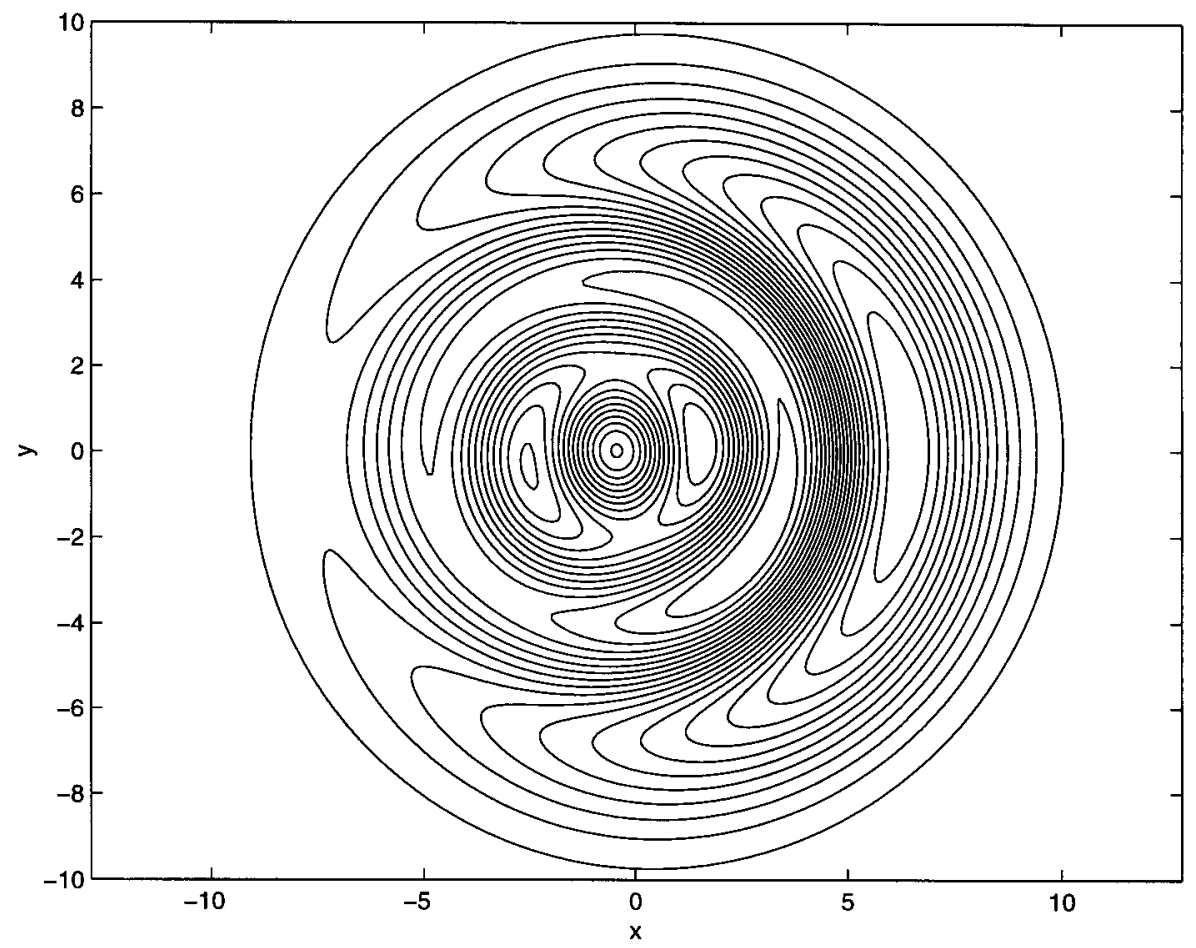

Рис. 14. Асимметричные магнитные поверхности при $x=0$ для равновесия плазмы (10.5)

ПримеР 6. Применим предыдушую конструкцию при $l=1, \beta=1 /\left(4 \gamma^{2}\right)$. При $N=0$ функция магнитного потока (10.4) принимает гауссов вид (7.21). При $N=1$ и $N=2$ уравнение (10.2) имеет только две пары целочисленных решений; поэтому функция магнитного потока (10.4) имеет вид (7.30). При $N=3$ диофантово уравнение (10.2) имеет три пары целочисленных решений $m=0, n=3, m=1, n=2$ и $m=2, n=0$. Из формул (10.3) следует $k_{3}=-2 / 3, k_{12}=-1 / 2$. Поэтому многочлен $B_{03}(x)(7.31)$ имеет вид $1-5 x+7 x^{2} / 2-x^{3} / 2$ и многочлен $B_{12}(x)$ есть $-3+9 x / 2-x^{2}$. Функция магнитного потока (10.4) принимает эквивалентньй вид

$$
\begin{aligned}
\psi= & e^{-\beta r^{2}}\left(1-10 \beta r^{2}+14 \beta^{2} r^{4}-4 \beta^{3} r^{6}\right. \\
& +a_{1} r\left(-3+9 \beta r^{2}-4 \beta^{2} r^{4}\right) \cos (z / \gamma-\phi) \\
& \left.+r^{2}\left(a_{2} \cos (2 z / \gamma-2 \phi)+b_{2} \sin (2 z / \gamma-2 \phi)\right)\right) .
\end{aligned}
$$

Рис. 14 показывает сечение $z=0$ магнитных поверхностей $\psi(r, z, \phi)=$ const при $a_{1}=2 / 5, a_{2}=-1 / 15, b_{2}=1 / 30, \beta=0.1$ и $\gamma=\sqrt{5 / 2}$. Функция $\psi(r, 0, \phi)(10.5)$ имеет два локальных максимума и три локальных минимума, которые определяют пять винтовых магнитных осей. Распределение кривых на рис. 14, очевидно, не обладает никакой симметрией, в то время как кривые на рис. 7 и 12 являются симметричными по отношению к отображению $y \rightarrow-y$. Обшие равновесия плазмы (10.4) не имеют никаких дополнительных симметрий. 
II. Другой класс точных равновесий плазмы сушествует при $\beta=p /\left(4 \gamma^{2}\right)$, где $p-$ нечетное число. Тогда $c_{1}=p / 2$ и диофантово уравнение (10.1) принимает вид

$$
2 p N=m^{2}+p m+2 p n .
$$

Это уравнение имеет $K+1$ пару целочисленных решений

$$
m=0, p, 2 p, \ldots, K p, \quad K=\left[\frac{\sqrt{8 N / p+1}-1}{2}\right], \quad n=N-\frac{m(m+p)}{2 p} .
$$

Коэффициенты $k_{N}(7.26)$ и $k_{m n}(7.29)$ суть

$$
k_{N}=\frac{1-\sqrt{8 N / p+1}}{2 N}, \quad k_{m n}=\frac{2 m / p+1-\sqrt{8 N / p+1}}{2 n} .
$$

Аналогично, соответствуюшие точные решения уравнения ДжфКО (7.5) имеют вид $(10.6)$

$$
\psi=e^{-\beta r^{2}}\left(a_{n} B_{0 N}(x)+\sum_{j=1}^{K} r^{j p} B_{j p . n}(x)\left(a_{j p . n} \cos (j p u / \gamma)+b_{j p . n} \sin (j p u / \gamma)\right)\right) .
$$

Эти функции магнитного потока определяют глобальные равновесия плазмы, которые линейно зависят от $2 K+1$ произвольных параметров $a_{N}, a_{j p . n}, b_{j p . n}$. Отметим, что параметр $m$ здесь является кратным нечетного числа $p: m=p, 2 p, \ldots, K p$. Следовательно, эти равновесия плазмы имеют дополнительные симметрии, так как они яВляются периодическими по переменной $z$ с периодом $2 \pi \gamma / p$ и по переменной $\phi \mathrm{c}$ периодом $2 \pi / p$.

III. Выведенные точные равновесия плазмы зависят от параметра $c_{1}=2 \beta \gamma^{2}$ довольно экзотическим образом. Для любого $c_{1}$ и любого целого числа $N$ существуют цилиндрически симметричные равновесия плазмы (7.24), (7.27). Для иррациональных $c_{1}$ эти равновесия не имеют точных равновесных возмущений с винтовой симметрией. Для любого рационального числа $c_{1}=p / q$ мы получаем из уравнения (10.1):

$$
n=N-\frac{m(q m+2 p)}{4 p} .
$$

Это выражение является целым числом, только если $m=r p$, где $r$ является целым, поскольку $p$ и $q$ взаимно просты. Максимально возможное целое $m$, удовлетворяющее уравнению (10.1), есть

$$
L=\left[\frac{p}{q}\left(\sqrt{\frac{4 q N}{p}+1}-1\right)\right]<2 \sqrt{\frac{p N}{q}} .
$$

Поэтому число $K$ целочисленных пар $(m, n)$, удовлетворяюших уравнению (10.1), есть

$$
K \leqslant \frac{L}{p}<2 \sqrt{\frac{N}{p q}}
$$


Следовательно, цилиндрически-симметричные равновесия (7.24), (7.27) для заданных рационального $c_{1}=p / q$ и целого $N$ не имеют точных равновесных возмущений с винтовой симметрией (7.30), если $p q>4 N$.

Если рациональное число $p / q$ является хорошей аппроксимацией некоторого иррационального $c_{1}$, то оба $p$ и $q$ являются большими, и поэтому из неравенства (10.7) следует, что диофантово уравнение (10.1) не имеет целочисленњых решений.

Для рациональных чисел $c_{1}=1 /(2 l)$ и $c_{1}=p / 2$ с нечетными $l$ и $p$ равновесия (7.24), (7.27) имеют точные равновесные возмущения с винтовой симметрией, которые зависят от $2 K+1$ параметров, где $K \approx \sqrt{2 N / l}$ и $K \approx \sqrt{2 N / p}$ соответственно.

\section{$\S 11$. Контрпримеры к теореме Паркера, обладающие винтовой симметрией}

I. Точное решение (7.24) определяет магнитное поле (7.7):

$$
\mathbf{B}_{N}(r)=\frac{\alpha \gamma \psi_{N}-r \psi_{N}^{\prime}}{r^{2}+\gamma^{2}} \widehat{\mathbf{e}}_{z}+\frac{\alpha r \psi_{N}+\gamma \psi_{N}^{\prime}}{r^{2}+\gamma^{2}} \widehat{\mathbf{e}}_{\phi}
$$

которое является ненулевым всюду в евклидовом пространстве $\mathbb{R}^{3}$. Действительно, предполагая выполненньм равенство $\mathbf{B}_{N}\left(r_{0}\right)=0$, мы получаем $\psi_{N}\left(r_{0}\right)=\psi_{N}^{\prime}\left(r_{0}\right)=0$, следовательно, $B_{0 N}\left(x_{0}\right)=B_{0 N}^{\prime}\left(x_{0}\right)=0$, что невозможно, так как все корни многочлена $B_{0 N}(x)$ являются простыми. Отсюда для любого $R$ и всех $r \leqslant R$ мы получаем $\left|\mathbf{B}_{N}(r)\right| \geqslant a_{N} B(R)>0$.

Пусть $\mathbf{B}_{N m n}(r, u)$ есть магнитное поле, определенное функцией магнитного потока $\psi_{N m n}(r, u)(7.30)$ и $A_{N}=\left(\left|a_{m n}\right|+\left|b_{m n}\right|\right) /\left|a_{N}\right|$. Внутри любой области $0 \leqslant r \leqslant R$ мы имеем

$$
\frac{\left|\mathbf{B}_{N m n}-\mathbf{B}_{N}\right|}{\left|\mathbf{B}_{N}\right|}<A_{N} \frac{C(R)}{B(R)}
$$

где $C(R)$ - некоторая гладкая функция. При $r \rightarrow \infty$ справедлива асимптотика

$$
\frac{\left|\psi_{N m n}-\psi_{N}\right|}{\left|\psi_{N}\right|}<A_{N}(2 \beta)^{n-N} \frac{2 C_{N m n}}{r^{2 N-2 n-m}}, \quad C_{N m n}=\frac{\left(1-n k_{m n}\right) N !}{\left(1-N k_{N}\right) n !} .
$$

Из неравенства $2 N>2 n+m$ следует, что $\left|\psi_{N m n}-\psi_{N}\right| /\left|\psi_{N}\right| \rightarrow 0$ при $r \rightarrow \infty$. Отсюда при $A_{N} \ll 1$ мы получаем $\left|\mathbf{B}_{N m n}-\mathbf{B}_{N}\right| /\left|\mathbf{B}_{N}\right| \ll 1$ всюду в $\mathbb{R}^{3}$.

Пусть $x_{N}$ есть максимальный корень многочлена $B_{0 N}(x)$. При $x>x_{N}$ мы имеем

$$
\left|B_{0 N}(x)\right|>\frac{1-N k_{N}}{N !}\left(x-x_{N}\right)^{N}, \quad\left|B_{m n}(x)\right|<\frac{1-n k_{m n}}{n !} x^{n} .
$$

Отсюда мы находим

$$
\frac{\left|\psi_{N m n}-\psi_{N}\right|}{\left|\psi_{N}\right|}<A_{N} C_{N m n} \frac{r^{m} x^{n}}{\left(x-x_{N}\right)^{N}} .
$$

Следовательно, при $x>N^{2 \kappa+1} x_{N}, 2 \kappa=\frac{\left|2 \log C_{N m n}-m \log (2 \beta)\right|}{(2 N-2 n-m) \log N}$, мы получаем

$$
\frac{\left|\psi_{N m n}-\psi_{N}\right|}{\left|\psi_{N}\right|}<A_{N}
$$


Такое же неравенство справедливо для магнитного поля. Таким образом, при $A_{N} \ll 1$ возмушения (7.30) могут быть значительными только при $x<N^{2 \kappa+1} x_{N}$. Подставляя $x=2 \beta l^{2}$, мы находим для характерного масштаба длины $l$ возмушений (7.30) по переменной $r$ :

$$
l \leqslant N^{\kappa} \sqrt{\frac{N x_{N}}{2 \beta}} .
$$

Неравенства (11.1), (11.2) означают, что равновесия плазмы (7.30) при $A_{N} \ll 1$ являются малыми возмущениями z-инвариантного равновесия (7.24) во всем евклидовом пространстве $\mathbb{R}^{3}$. Отсюда мы получаем, что условие Паркера, что "локальное возмущение поля является мальм по сравнению с полным полем" [23; с. 361], вьполнено всюду. Условие Паркера, что “магнитное поле является аналитическим в его отклонении є от инвариантного поля $B_{i}(x, y)$ " [23; с. 378], выполнено, поскольку точные решения (7.30) являются линейными функциями малых параметров $a_{m n}, b_{m n}$. Условие Паркера, что длина потока $L$ является "большой сравнительно с характерным трансверсальным.м масштабом вариачии l поля" [23; с. 362], удовлетворено, поскольку $l \leqslant N^{\kappa} \sqrt{N x_{N} /(2 \beta)}$ и длина трубки потока $L$ может быть выбрана произвольно большой для $z$-инвариантного равновесия (7.30). Следовательно, $L \gg l$. Все возмущения (7.30) не являются $z$-инвариантньми. Поэтому равновесия плазмы (7.30) являются контрпримерами к теореме Паркера.

Точные равновесия плазмы (10.4) и (10.6) доставляют семейства контрпримеров к теореме Паркера, которые зависят от $2 K+1$ произвольных параметров, где $K \geqslant 1-$ произвольное целое число.

\section{§ 12. Заключение}

В ч. I мы представили два семейства точных осесимметричных решений уравнений равновесия плазмы, см. формулы (2.15) и (5.1). В силу изоморфизма между уравнениями (1.1) и (1.2) семейство (2.15) описьвает также глобальные равновесия идеальной несжимаемой жидкости в $\mathbb{R}^{3}$. Эти равновесные решения зависят от произвольного числа параметров, которые могут быть выбраны таким образом, что топология магнитного поля становится произвольно сложной. Поля определяются комбинациями вложенных цилиндрических магнитных поверхностей и вложенных тороидальных магнитных поверхностей. Обшие решения в семействе (2.15) являются квазипериодическими по $z$, что означает, что магнитные силовые линии никогда не повторяются в z-направлении, однако могут иметь структуру, произвольно близкую к начальньм данным. Их “характер вращения" меняется непрерывно вместе с $z$ и не повторяется.

Равновесные решения $(2.15),(5.1),(5.4)$ являются глобальными и всюду гладкими. Они не имеют разрывов и не имеют поверхностных токов. Эти решения моделируют разнообразные магнитные явления, которые наблюдались в астрофизических струях (в сопутствуюшей системе отсчета) и в солнечной корональной плазме. Квазипериодическое поведение, которое является эргодическим по $z$, доставляет наилучшее возможное аналитическое описание пространственного распределения магнитного поля и электрического тока в астрофизических струях.

Каждое из этих семейств, (2.15) и (5.4), образует контрпримеры к теореме Паркера, которая была сформулирована более 25 лет назад и была отправной точкой боль- 
шого числа работ, опубликованных за это время. Наиболее недавней работой, которая использует теорему Паркера существенным образом, является статья [48] 1996 года.

В части II мы представили точные равновесия плазмы (7.30) с винтовой симметрией, которые удовлетворяют уравнению ДжфКО (7.5). Решения (7.30) описывают также глобальные магнитогидродинамические равновесия (1.5) для параллельных векторных полей $\mathbf{B}=\lambda \mathbf{V}, \lambda \neq \sqrt{\rho \mu}$. Магнитные поля определяются комбинациями вложенных и винтовым образом вращающихся цилиндрических магнитных поверхностей. Для равновесий (7.30) общие магнитные силовые линии являются квазипериодическими по $z$, что означает, что магнитные силовые линии никогда не повторяются в z-направлении, но могут иметь структуру, произвольно близкую к начальным данным. Их "характер вращения" непрерывно изменяется вместе с переменной $z$ и не повторяется.

Равновесные решения (7.24), (7.30) являются глобальными и всюду гладкими. Они не имеют разрывов и не имеют слоев тока. Эти решения моделируют различные магнитные явления, которые наблюдались в токамаках (внешние и внутренние кинкмоды), в астрофизических струях (струя в эллиптической галактике Вирго А или Мессиер 87) и в солнечных протуберанцах.

Полученные точные равновесия плазмы (7.30), (10.4) и (10.6) образуют контрпримеры к теореме Паркера, обладающие винтовой симметрией.

Точные решения (10.4) и (10.6) для общего $r=r_{0}$ имеют вид рядов Фурье по переменной $u$. Поэтому эти решения при общих $r=r_{0}$ приближают любую гладкую функцию $\psi\left(r_{0}, u\right)$, которая является $2 \pi \gamma$-периодической по $u$ для решений $(10.4)$ и $2 \pi \gamma / p$-периодической по $u$ для (10.6). Вследствие этого точные решения (10.4) и (10.6) описьвают довольно обшие глобальные равновесия плазмы, обладающие винтовой симметрией.

\section{СПИСОК ЛИТЕРАТУРЫ}

[1] Bogoyavlenskij O. I. Astrophysical jets as exact plasma equilibria // Phys. Rev. Lett. 2000. V. 84. P. 1914-1917.

[2] Bogoyavlenskij O. I. Counterexamples to Parker's theorem // J. Math. Phys. 2000. V. 41. P. 2043-2057.

[3] Bogoyavlenskij O. I. Exact helically symmetric plasma equilibria // Lett. Math. Phys. 2000 (to appear).

[4] Grad H., Rubin H. Hydromagnetic equilibria and force-free fields // Proceedings of the Second United Nations International Conference on the Peaceful Uses of Atomic Energy. V. 31. Geneva: United Nations, 1958. P. 190-197.

[5] Шафранов В. Д. О магнитогидродинамических равновесных конфигурациях // ЖЭЭФ. 1958. T. 6. C. $545-554$.

[6] Kruskal M. D., Kulsrud R. M. Equilibrium of a magnetically confined plasma in a toroid // Phys. Fluids. 1958. V. 1. P. 265-274.

[7] Spitzer L. The stellarator concept // Phys. Fluids. 1958. V. 1. P. 253-264.

[8] Chandrasekhar S. Axisymmetric magnetic fields and fluid motions // Astrophys. J. 1954. V. 124. P. 232-243.

[9] Chandrasekhar S., Prendergast K.H. The equilibrium of magnetic stars // Proc. Nat. Acad. Sci. U. S. A. 1956. V. 42. P. 5-9.

[10] Biskamp D. Nonlinear Magnetohydrodynamics. Cambridge: Cambridge Univ. Press, 1993.

[11] Browning P. K. Magnetohydrodynamics in solar coronal and laboratory plasmas: a comparative study // Phys. Rep. 1988. V. 169. P. 329-384.

[12] Freidberg J. F. Ideal Magnetohydrodynamics. New York: Plenum Press, 1987. 
[13] Кадомцев Б. Б. Равновесия плазмы с винтовой симметрией // ЖЭТФ. 1960. Т. 10. C. $962-963$.

[14] Соловьев Л. С. Теория гидродинамической устойчивости тороидальных плазменных конфигураций // ЖЭТФ. 1968. Т. 26. С. 400-407.

[15] Herrnegger F. On the equilibrium and stability of the belt pinch // Proceedings of V European Conference on Controlled Fusion and Plasma Physics. V. I. Grenoble, 1972. P. 26.

[16] Luc H., Maschke E. K., Touche J. Tokamak equilibria with high $\beta$ poloidal // Proceedings of V European Conference on Controlled Fusion and Plasma Physics. V. I. Grenoble, 1972. P. 23.

[17] Maschke E. K. Exact solutions of the MHD equilibrium equation for a toroidal plasma // Plasma Phys. 1973. V. 15. P. 535-541.

[18] Lundquist S. Studies in magneto-hydrodynamics // Ark. Fys. 1952. V. 5. P. 297-347.

[19] Proudman J. On the motion of solids in a liquid possessing vorticity // Proc. Roy. Soc. London. 1916. V. 92. P. 408-424.

[20] Taylor G. I. Motion of solids in fluids when the flow is not irrotational // Proc. Roy. Soc. London. 1917. V. 93. P. 99-113.

[21] Taylor G. I. Experiments with rotating fluids // Proc. Roy. Soc. London. 1922. V. 100. P. 114-121.

[22] Parker E. N. Topological dissipation and the small-scale fields in turbulent gases // Astrophys. J. 1972. V. 174. P. 499-510

[23] Parker E. N. Cosmical Magnetic Fields. Oxford: Clarendon Press, 1979.

[24] Parker E. N. Spontaneous Current Sheets in Magnetic Fields. Oxford: Oxford Univ. Press, 1994.

[25] Massaglia S., Bodo G. (Eds.) Astrophysical Jets. Open Problems. Amsterdam: Gordon and Breach, 1998.

[26] Hardy G. H., Wright E. M. An Introduction to the Theory of Numbers. Oxford: Clarendon Press, 1998.

[27] Захаров В. Е., Шабат А. Б. Точная теория двумерной самофокусировки и одномерной самомодуляции волн в нелинейных средах // ЖЭТФ. 1972. Т. 34. С. 62-69.

[28] Ablowitz M.J., Kaup D. J., Newell A.C., Segur H. Method for solving the sine-Gordon equation // Phys. Rev. Lett. 1973. V. 30. P. 1262-1264.

[29] Bogoyavlenskij O.I. Extended integrability and bi-Hamiltonian systems // Comm. Math. Phys. 1998. V. 196. P. 19-51.

[30] Goeler S., Stodiek W., Sauthoff N. Studies of internal disruptions and $m=1$ oscillations in tokamak discharges with soft- $X$-ray techniques // Phys. Rev. Lett. 1974. V. 33. P. 1201-1203.

[31] Кадомцев Б. Б., Погутце О. П. Нелинейные винтовые возмущения плазмы в токамаке // ЖЭТФ. 1974. Т. 38. С. 283-290.

[32] Schank D. D., Garamana E. J., Nebel R. A. Three-dimensional magnetohydrodynamic studies of the reversed-field pinch // Phys. Fluids. 1985. V. 28. P. 321-333.

[33] Ferrari A. Modeling extragalactic jets // Annual Rev. Astron. Astroph. 1998. V. 36. P. 539-598.

[34] Blandford R. D., Payne D. G. Hydromagnetic flows from accretion discs and the production of radio jets // Monthly Notices Roy. Astronom. Soc. 1982. V. 199. P. 883-903.

[35] Burbridge G. R. On synchrotron radiation from Messier 87 // Astrophys. J. 1956. V. 124. P. 416-429.

[36] Hines D. C., Owen F. N., Eilek J. A. Filaments in the radio lobes of M87 // Astrophys. J. 1989. V. 347. P. 713-726.

[37] Russell C. T., Priest E. R., Lee L. C. (Eds.) Physics of Magnetic Flux Ropes. Washington, DC: Amer. Geophys. Union, 1990.

[38] Johnson J. L., Oberman C. R., Kulsrud R. M., Frieman E. A. Some stable hydromagnetic equilibria // Phys. Fluids. 1958. V. 1. P. 281-296.

[39] Mordell L. J. Diophantine Equations. London: Academic Press, 1969.

[40] Low B. C. Exact static equilibrium of vertically oriented magnetic flux tubes // Solar Phys. 1980. V. 67. P. 57-77. 
[41] Tsinganos K. Magnetohydrodynamic equilibrium. IV. Nonequilibrium of nonsymmetric hydrodynamic topologies // Astrophys. J. 1982. V. 259. P. 832-843.

[42] Yu G. Hydrostatic equilibrium of hydromagnetic fields // Astrophys. J. 1973. V. 181. P. $1003-1008$.

[43] Low B. C. On magnetostatic equilibrium in a stratified atmosphere // Solar Phys. 1980. V. 65. P. $147-165$.

[44] Rosner R., Knobloch E. On perturbations of magnetic field configurations // Astrophys. J. 1982. V. 262. P. 349-357.

[45] Tsinganos K. C., Distler J., Rosner R. On the topological stability of magnetostatic equilibria // Astrophys. J. 1984. V. 278. P. 409-419.

[46] Vainstein S. I., Parker E. N. Magnetic nonequilibrium and current sheet formation // Astrophys. J. 1986. V. 304. P. 821-827.

[47] Villata M., Tsinganos K. Exact solutions for helical magnetohydrodynamics equilibria // Phys. Fluids B. 1993. V. 5. P. 2153-2164.

[48] Low B. C. The spontaneous formation of current-sheets in astrophysical magnetic fields // Solar and Astrophysical Magnetohydrodynamic Flows. Dordrecht: Kluwer, 1996. P. 109-131.

[49] Van Ballegooijen A. A. Electric currents in the solar corona and the existence of magnetostatic equilibrium // Astroph. J. 1985. V. 298. P. 421-430.

[50] Van Ballegooijen A. A. Cascade of magnetic energy as a mechanism of coronal heating // Astroph. J. 1986. V. 311. P. 1001-1014.

[51] Bragg S. L., Haw thorne W. R. Some exact solutions of the flow through annular cascade actuator discs // J. Aeronaut. Sci. 1950. V. 17. P. 243-249.

[52] Krat V.A., Osherovich V.A. On the excited states of magnetic configurations in connection with the characteristic properties of sunspots // Solar Phys. 1976. V. 50. P. 65-68.

[53] Osherovich V. A. A new magneto-hydrostatic theory of sunspots // Solar Phys. 1982. V. 77. P. $63-68$.

[54] Osherovich V. A., Lawrence J. K. Elaboration of the new magnetohydrostatic sunspot theory (double return flux model) // Solar Phys. 1983. V. 88. P. 117-135.

[55] Szego G. Orthogonal Polynomials. Providence, RI: Amer. Math. Soc., 1967.

[56] Courant R., Hilbert D. Methods of Mathematical Physics. New York: Interscience Publishers, 1953.

[57] Abramowitz M., Stegun I. A. Handbook of Mathematical Functions with formulas, graphs and mathematical tables. Washington, D. C.: V. S. Department of Commerce, National Bureau of Standards, 1964.

[58] Ribenboim P. Algebraic Numbers. New York: Wiley-Interscience, 1972.

[59] Чандрасекхаран К. Арифметические функции. М.: Наука, 1975.

[60] Apostol T. M. Introduction to Analytic Number Theory. New York: Springer-Verlag, 1976. 